Mitteilungen der Österreichischen Geographischen Gesellschaft, 161. Jg., S. 35-62

(Annals of the Austrian Geographical Society, Vol. 161, pp. 35-62)

Wien (Vienna) 2019, https://doi.org/10.1553/moegg161s35

\title{
Die Sozialräumliche STrukTur Der LEADER-Region Vorderland-Walgau-Bludenz - Auf Dem Weg zUR alpinen Postsuburbia?
}

\author{
Johannes HeRBURGER, Vaduz* \\ Erste Einreichung / initial submission: 05/2018; revidierte Fassung / revised submission: 11/2018; \\ endgültige Annahme / final acceptance: 01/2019
}

mit 7 Abb. und 4 Tab. im Text

\section{INHALT}

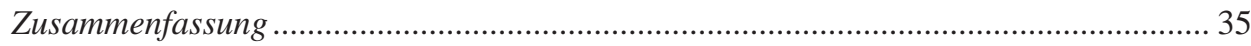

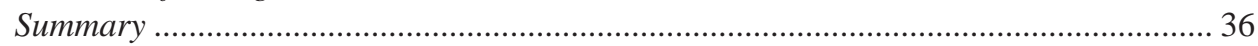

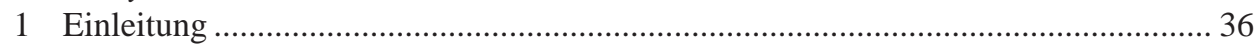

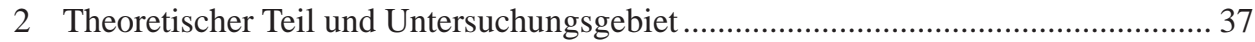

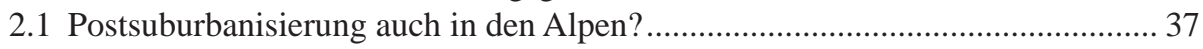

2.2 Die LEADER-Region Vorderland-Walgau-Bregenz ........................................ 41

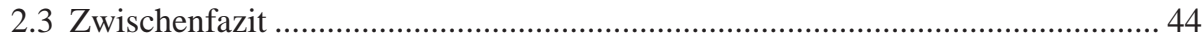

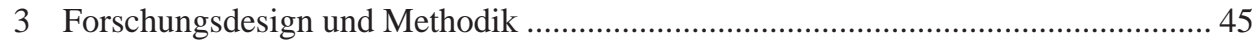

4 Der Weg zur Raumtypisierung - Indikatoren und Analyse..................................... 47

5 Sozialräumliche Typisierung der LEADER-Region Vorderland-Walgau-Bregenz ... 51

6 Und was nun? - Handlungsempfehlungen als Ergebnis der sozialräumlichen

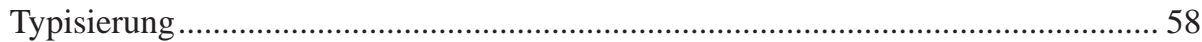

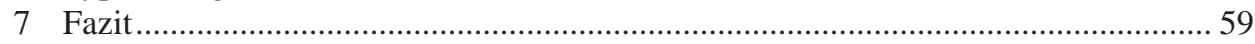

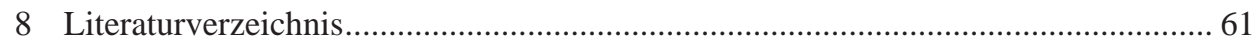

\section{Zusammenfassung}

In der vorliegenden Studie wird die sozialräumliche Struktur der LEADER-Region Vorderland-Walgau-Bludenz im österreichischen Bundesland Vorarlberg vor dem theoretischen Hintergrund der Konzepte von Suburbanisierung und Postsuburbanisierung untersucht. Auf Basis dieser theoretischen Fundierung wurden 13 Indikatoren auf Basis von 250 × 250 m Bevölkerungsrasterdaten der Bundesanstalt Statistik Austria abgeleitet, deren Wechsel-

\footnotetext{
* Johannes Herburger MA BA, Institut für Architektur und Raumentwicklung, Universität Liechtenstein, Fürst-Franz-Josef-Straße, FL-9490 Vaduz, Liechtenstein; Preisträger des Förderungspreises der Österreichischen Geographischen Gesellschaft 2016. E-Mails: johannes.herburger@uni.li, https://orcid.org/0000-00034792-182X.
} 
wirkungen mittels Hauptkomponenten- und Clusteranalyse untersucht wurden. Dadurch konnten sieben Raumtypen bestimmt werden, welche von hochverdichteten Ortszentren über postsuburbane und suburbane Quartiere bis hin zur sozialen und ethnischen Segregation bestimmter Bevölkerungsgruppen reichen. Generell zeigt sich, dass der demographische und gesellschaftliche Wandel alle Raumtypen vor weitreichende Veränderungen stellt und insbesondere der Raumtyp „,Postsuburbia “ vor einer dynamischen Entwicklung steht, die einer hohen raumordnerischen und politischen Beachtung bedarf. Ebenso spiegelt sich der Druck auf die Siedlungsränder entgegen allen raumordnerischen Zielsetzungen klar in den Ergebnissen wider.

Schlagwörter: Suburbanisierung, Postsuburbanisierung, Alpen, Sozialraumanalyse, Raumplanung, Vorarlberg, LEADER-Region Vorderland-Walgau-Bludenz

\section{Summary}

\section{The Socio-Spatial Structure of the LEADER-Region Vorderland- Walgau-Bludenz - ON THE Way to AN Alpine Postsuburbia?}

This paper deals with the socio-spatial structures of the LEADER-Region Vorderland-Walgau-Bludenz in the Austrian federal state (Bundesland) of Vorarlberg. From the theoretical basis of suburbanisation and postsuburbanisation 13 indicators, based on population data on $250 \times 250 \mathrm{~m}$ grid level, are derived and analysed through principal-component and cluster analysis. This analysis classified seven social-area types with specific characteristics that range from town centres to suburban and postsuburban quarters and neighbourhoods which represent social and ethnical segregation. The overall result of this social-area typification is that social and demographic change implies a lot of future challenges for all social-area types. This is especially true for the postsuburban social areas, which face a dynamic future needing much consideration from spatial planning. Furthermore the pressure on the edges of the settlements is ongoing, contradicting all objectives of special planning.

Keywords: Suburbanisation, postsuburbanisation, social area analysis, Austrian Alps, spatial planning, Vorarlberg, LEADER-Region Vorderland-Walgau-Bludenz

\section{Einleitung}

Unsere Gesellschaft wird komplexer. Unsere Raumstrukturen werden fragmentierter und differenzierter. Was passiert, wenn man diese beiden Prozesse in integrierter Form betrachtet? Insbesondere in den wirtschaftlich starken Stadtregionen Europas entwickelte sich im Umfeld der Kernstädte über die Jahrzehnte hinweg eine gänzlich neue Raumstruktur, die weder dem Dualismus von Stadt und Land noch der klassischen Suburbia mit ihren Einfamilienhaussiedlungen entspricht. Es entstehen neue Wohnformen und neue Bevölkerungsgruppen ziehen hin. Parallel dazu vollzieht sich ein gesellschaftlicher und demographischer Wandel, der alle Ebenen der Politik und Verwaltung vor völlig neue Herausforderungen stellt. 
Das Ziel dieser Arbeit ist die Aufschlüsselung der durch diese Prozesse entstehenden sozialräumlichen Strukturen in der LEADER-Region Vorderland-Walgau-Bludenz in Vorarlberg. Die Region kann als ursprünglich kleinstädtisch-dörflich geprägtes Gebiet mit den Zentren Feldkirch und Bludenz charakterisiert werden, dem eine Kernstadt, wie sie in den klassischen Modellen angenommen wird, jedoch fehlt. Aufgrund dieser Ausgangssituation und der weiteren siedlungshistorischen Entwicklung, getragen von der nachkriegszeitlichen Industrialisierung und Suburbanisierung, sind spezifische sozialräumliche Strukturen entstanden. Vor dem Hintergrund, dass die Städte und Stadtregionen in den Alpen größtenteils nur von geringem Forschungsinteresse in der Stadt- bzw. Umlandforschung sind (PERLIK 2001, S. 13), soll mit diesem Beitrag auch diskutiert werden, inwiefern das historisierte und romantisierte Bild der Alpenstädte überholt ist.

Zur Beantwortung der Forschungsfrage „Welche sozialräumlichen Strukturen lassen sich in der LEADER-Region Vorderland-Walgau-Bludenz klassifizieren?" wird im ersten Teil die Theorie zur Suburbanisierung und Postsuburbanisierung vorgestellt und diskutiert, inwiefern diese auch auf eine alpine Stadtregion ohne Kernstadt anwendbar ist, wodurch weitere Fragestellungen operationalisiert werden können. Anhand dieser theoretischen Fundierung wird darauf aufbauend ein Indikatorenset entwickelt, das auf den Bevölkerungsrasterdaten der Bundesanstalt Statistik Austria im Ausmaß von 250 x 250 m basiert. Dadurch können kleinräumige Muster und Strukturen aufgezeigt werden, die mittels Hauptkomponenten- und Clusteranalyse in eine Raumtypisierung münden. Diese Raumtypisierung soll spezifische sozialräumliche Strukturen und Problemfelder in der Region aufdecken, anhand derer Handlungsempfehlungen artikuliert werden können.

\section{Theoretischer Teil und Untersuchungsgebiet}

\subsection{Postsuburbanisierung auch in den Alpen?}

Der Startschuss für die Suburbanisierung im heutigen Sinn kann auf die 1950er Jahre datiert werden. Durch die Festigung der europäischen Volkswirtschaften und das damit in Zusammenhang stehende ökonomische Wachstum kam es zu einer breiten Zunahme des Wohlstands, wodurch auch die Voraussetzungen für ein Mobilitäts- und Wohnflächenwachstum gesetzt wurden (ARING 2001, S. 2; FASSMAnn 2009, S. 183). Dadurch wurde das Einfamilienhaus mit Garten folgerichtig zum sichtbarsten Symbol der Suburbanisierung, welches „,seit gut 100 Jahren die Entwicklung unserer politischen, sozialen wie eben räumlichen Gesellschaftsformen so maßgeblich mitbestimmt" (SAIKO 2003, S. 95). Unterstützt wurde dieser in der Bevölkerung geweckte Wunsch durch die relativ niedrigen Grundstückspreise in der Siedlungsperipherie und die gute Erschließung durch öffentliche und private Verkehrsformen. Die finanzielle Förderung des Eigenheimbaus durch die öffentliche Hand in direkter oder indirekter Form trug ihr Übriges dazu bei (BorSDORF 2009, S. 9; FASSMANn 2009, S. 183).

Dadurch entstanden hinsichtlich der Bevölkerungsstruktur homogene Siedlungsräume, die primär von jungverheirateten Paaren in der Expansionsphase ihres Lebenszyklus mit mittlerem bis hohem Einkommen bewohnt wurden (HeLBICH 2008, S. 11, zitiert nach 
FRIEDRICHS 1983). Bis in die 1980er Jahre konnte somit eine größtenteils klare Struktur erkannt werden, in welcher der Stadtkern überwiegend alle essenziellen zentralörtlichen Funktionen innehatte und sich die Suburbia im Gegensatz dazu hauptsächlich als Schlafstadt auszeichnete (BORSDORF und BENDER 2010, S. 146).

Zusätzlich zur Wohn-Suburbanisierung folgten dieser Randwanderung bald auch der Einzelhandel und andere Branchen, wodurch sich am ehemaligen Siedlungsrand genuine Arbeitsplatzzentren entwickelten (BRAKE et al. 2005, S. 85ff; BORSDORF und BENDER 2010, S. 146). Von staatlicher Seite gesteuert und gefördert wurde dies durch den Ausbau eines immer leistungsfähigeren Individualverkehrsnetzes aus Autobahnen und Schnellstraßen, das neben der Verbindung von zentralen Orten auch die Standortpotenziale von Kleingemeinden gänzlich veränderte (GöRGL 2008, S. 15). Zusammen mit den prägenden Prozessen der Globalisierung und des Postfordismus entwickelte sich in weiterer Folge ein neues, multizentrales, räumliches Arrangement von spezialisierten, oftmals schon von der Kernstadt emanzipierten Zentren, die wichtige Aufgaben in der Stadtregion übernommen haben (Helbich 2008: S. 16; GöRGL 2008, S. 15). „Nicht Frankfurt, München, Lyon oder Lissabon stehen im internationalen Wettbewerb, sondern die polyzentrischen Stadtregionen, die sich um diese Städte herum entwickelt haben, mit einer Vielzahl von kleinen und mittleren Städten, die sich für Unternehmen [...] als identifizierbare Siedlungskerne darstellen“ (KunZMANN 2001, S. 213f).

Die Entstehung dieses polyzentralen räumlichen Arrangements bezieht sich, wie am Zitat von KUnZMANN sichtbar wird, primär auf Großstadtregionen. Doch auch innerhalb der Alpen kam es zu ähnlichen Veränderungen der räumlichen Strukturen, wie sie an den Rändern der Großstadtregionen sichtbar wurden. Die rasant steigende Mobilität infolge der Massenmotorisierung, des Straßenbaus und des billigen Erdöls spielte auch in den Alpen eine essenzielle Rolle für die rasche Ausbreitung aller städtischen Funktionen in die Fläche: Beginnend beim Wohnen über den Einzelhandel bis hin zu Industrie und Dienstleistungen (BätzING 2015, S. 203ff). Befördert wird diese scheinbar diffuse und ungesteuerte Entwicklung in Österreich und Deutschland durch die starke Stellung der lokalen Selbstverwaltung im Bereich der örtlichen Raumordnung, die den Kommunen eine mächtige Position einräumt (GörGL 2008, S. 16f).

Aus siedlungsstruktureller Sicht unterscheiden sich die heutigen Entwicklungen innerhalb der alpinen Stadtregionen also kaum von den postsuburbanen Entwicklungen in den Großstadtregionen. Deutlich ist allerdings ein Auslassen einer wichtigen Epoche der Urbanisierung, denn durch die einstige Peripherisierung der Alpen, die sich vor allem durch die Bildung der Nationalstaaten und die damit einhergehende Grenzziehung im 19. Jahrhundert ergab, war auch der ,impact“ der Industrialisierung in dieser Makroregion sehr gering (PERLIK et al. 2001, S. 245). So sind klassische Siedlungselemente der Industrialisierung des späten 19. und beginnenden 20. Jahrhunderts wie Industriegebiete, Arbeiterwohnsiedlungen oder gründerzeitliche Villenviertel in den Alpen kaum bis gar nicht vorhanden (B̈̈TZING 2015, S. 203).

Die Aufwertung der Nord-Süd-Achsen durch den Ausbau der großen Transit-Verkehrswege infolge des geteilten Europa nach dem Zweiten Weltkrieg begünstigte schließlich die gestiegene inneralpine Erreichbarkeit (BäTZING 2015, S. 204f), wodurch die Alpenstädte jedoch stark von den wirtschaftsstrukturellen Prozessen der perialpinen Metropolregionen 
abhängig wurden (PERLIK 2001, S. 172). Durch diese neue wirtschaftliche Entwicklung wird auch die Bevölkerungsveränderung in den Alpen beeinflusst. 1980 lag das Bevölkerungswachstum erstmals über dem europäischen Durchschnitt und stieg bis heute weiter an (BÄTZING 2015, S. 206).

Trotz fehlender großstädtischer Kernräume wurden die alpinen Stadtregionen also voll in die Suburbanisierungsbewegungen einbezogen. Einen wesentlichen Einfluss übt des Weiteren das Relief aus, das die Siedlungsentwicklung entlang eines zusammenhängenden Bandes auf dem Talboden fördert, da nur dort die Erreichbarkeit optimal ist (BÄTzING 2015, S. 204f). Die Entwicklungsmuster der außeralpinen Metropolen dehnten sich also auf den alpinen Raum aus.

Die zunehmende wirtschaftliche Ausdifferenzierung und Entwicklung innerhalb der alpinen und außeralpinen Stadtregionen hat dazu geführt, dass nun sowohl hoch- als auch niedrigqualifizierte Arbeitsstellen im sekundären und tertiären Sektor verfügbar sind. Zusammen mit dem Einfluss des staatlich geförderten Wohnbaus konnte sich nun auch die alpine Postsuburbia als Wohn- und Arbeitsort für alle Bevölkerungsschichten etablieren, was sich in der Heterogenisierung und Pluralisierung der Lebensstile sowie einer gestiegenen baulichen und architektonischen Vielfalt widerspiegelt. Aus diesem Grund wird in den folgenden Abschnitten noch weiter erläutert, wie sich sowohl die demographische, sozio-ökonomische und sozio-kulturelle als auch die bauliche und siedlungsstrukturelle Konstellation der (Post-)Suburbia im Allgemeinen und der alpinen Postsuburbia im Speziellen verändert haben.

\section{De-Agrarisierung und ihre Folgewirkungen}

Unter der De-Agrarisierung sind nicht nur der landwirtschaftliche Strukturwandel und die volkswirtschaftlich geringere Bedeutung der Landwirtschaft zu verstehen, sondern deren gesamte Auswirkungen auf den physiognomisch-funktionalen Wandel der Siedlungsstruktur, der auch durch die Intensivierung der Landwirtschaft und den dadurch geringeren Flächenbedarf gefördert wurde: Neue Gebäude werden errichtet, eine neue Vielfalt der Architekturstile ist festzustellen, die Haushaltsstruktur tendiert zu kleineren Einheiten bis hin zum Single-Haushalt und neue ,Lifestyles“ haben die Ausweitung der Freizeitinfrastruktur zur Folge (BorsDorf 2006, S. 85). Der strukturelle Wandel des Agrarsektors und die Expansion des Industrie- und Dienstleistungssektors sind, wie oben bereits angemerkt wurde, in den Alpen ebenfalls seit Jahrzehnten zu beobachten (PERLIK et al. 2001, S. 243).

Allerdings muss bedacht werden, dass der Bedeutungsverlust der Landwirtschaft sowohl innerhalb als auch außerhalb der Alpen - bereits vor den eigentlichen Suburbanisierungsbewegungen angebrochen ist und sich lediglich die sozialen und kulturellen Umbrüche (Abnahme der Kirchenbesuche, Entwicklung der Konsumorientierung etc.) erst in den 1960er Jahren einstellten. In der Folge entstanden suburbane Wohnorte oder gewerblich geprägte Vororte aus den einstigen Dörfern (Trossbach und ZimmermanN 2006, S. 256).

Diese Prozesse führten und führen in der Suburbia zu sich ausdifferenzierenden sozio-ökonomischen, sozio-kulturellen und sozio-demographischen Strukturen in der Be- 
völkerung, wobei auch die Postsuburbia über Quartiere verfügt, in denen das familienzentrierte Modell des Eigenheims, mit Anschluss an Landschaft und Autobahn, immer noch das höchste soziale Gut darstellt (PrigGe 1998, S. 10). Es sind aber nicht mehr nur die gut ausgebildeten und gut verdienenden Mittelschichtfamilien, die in diese Räume ziehen. Das Spektrum der Bewohnerinnen und Bewohner ist heute sehr diversifiziert: Es reicht von Familien mit stark überdurchschnittlichem Haushaltseinkommen über junge Zweipersonenhaushalte, die auch potenzielle Gentrifizierer sein könnten, bis zu sozial schwächeren Gruppen (Aring und Herfert 2001, S. 51).

\section{Die Alterung der Bevölkerung - auch in Suburbia?}

Generell wird der Prozess der Alterung einer Bevölkerung durch zwei sich gegenseitig verstärkende Prozesse vorangetrieben. Einerseits ist dies die langfristige Abnahme der Gesamtfertilitätsrate (B̈̈HR 2010, S. 85), wobei der Alterungsprozess andererseits auch durch die steigende Lebenserwartung stetig vorangetrieben wird. Insbesondere dadurch wächst die Zahl der Hochbetagten, also der Personen, die ein Alter von über 85 Jahren erreichen, laufend (PoHL 2010, S. 196).

In diesem Zusammenhang konnten NuissL und BigALKe (2007) zeigen, dass das Wohnen in suburbanen Quartieren von den dortigen Bewohnern als positiv empfunden wird und auch deren soziale Kontakte nicht nur auf das nähere Wohnumfeld fokussiert sind. „Vielmehr darf es als Ausdruck der generellen Enträumlichung sozialer Beziehungen [...] gelten, dass die zwischenmenschlichen Beziehungen auch älterer Suburbanitinnen und Suburbaniten keineswegs allein an deren unmittelbares Wohnumfeld gebunden sind“" (ebd., S. 389). Dies ist allerdings nur solange der Fall, wie die Unterausstattung mit sozialen Infrastrukturen durch die hohe selbstständige Mobilität kompensiert werden kann. Sollte diese nicht mehr gewährleistet sein, werden die nachbarschaftlichen Beziehungen zur Vorbeugung gegen die soziale Isolation immer wichtiger. Dazu gesellen sich noch infrastrukturelle Problemfelder, die mit der Bausubstanz und Behindertengerechtigkeit der Gebäude sowie dem näheren Wohnumfeld zusammenhängen (SPELLERBERG und GERHARDS 2013, S. 137).

Konträr zur Zunahme der älteren Bevölkerungsgruppen kommt es aufgrund der sinkenden Geburtenzahlen zu einer stetigen Abnahme junger Bevölkerungsgruppen. Die anteilsmäßig stärkste Alterskohorte sind die Personen, die zwischen 1965 und 1969 geboren wurden. Diese Zeit kann auch als „hohe Zeit des Babybooms“ betrachtet werden, denn vor allem ab den 1970er Jahren setzte mit der Abnahme der Fertilitätsraten der sogenannte „Baby Bust“ ein (Lesthaeghe 2011, S. 181). Die Gründe für das Absinken der Geburtenraten sind vielfältig und hängen sehr eng mit einem Wandel gesellschaftlicher Werte und Einstellungen zusammen (Rolle von Frau und Mann, Scheidungen, individualisierte Lebensformen, etc.). Oftmals wird hierbei vom sogenannten Pillenknick gesprochen, was allerdings eindeutig zu kurz greift. Das Aufkommen von Verhütungsmitteln spielt zwar mit eine Rolle, kann aber eher als Katalysator dieser Entwicklung bezeichnet werden (BÄHR 2010, S. 195).

Wie anhand dieser Phänomene sichtbar wird, laufen diese parallel zur De-Agrarisierung und sind somit auch ein Merkmal der gesellschaftlichen Ausdifferenzierung, die auch die Entwicklung der Postsuburbia beeinflusst. Nichtsdestotrotz muss darauf hingewiesen werden, dass Regionen mit kontinuierlichem Zuzug von jungen Personen nicht unmittel- 
bar bzw. erst in Zukunft vom Alterungsprozess betroffen sind, wobei es insbesondere bei kleinräumig differenzierter Betrachtung solcher Regionen zu unterschiedlichen Mustern kommen kann (PoHL 2010, S. 197ff; BRAKE et al. 2005, S. 20).

\section{Wanderung nach Suburbia - auch ohne Umweg}

Die ökonomisch motivierte internationale Zuwanderung wird aufgrund des eben beschriebenen Alterungsprozesses gerade für wirtschaftlich prosperierende Regionen zukünftig ein wichtiger Faktor für das Bevölkerungswachstum sein (BRAKE et al. 2005, S. 73). Aufgrund des diversifizierten Arbeitsplatzangebots bezieht sich die Zuwanderung mittlerweile auf alle sozio-ökonomischen Schichten und somit auch auf sozial schwächere Migrantinnen und Migranten (Aring und Herfert 2001, S. 51). Der Status von Zuwanderern in postsuburbane Regionen wird also nach Merkmalen ihrer sozialen Lage und insbesondere auch in Hinblick auf den Ausländeranteil immer heterogener, die Selektivität nimmt ab, die soziale Polarisierung jedoch zu (vgl. BrAKe et al. 2005, S. 20ff).

Gerade hierbei sollte nochmals darauf verwiesen werden, dass in der europäischen Suburbia schon in den 1960er Jahren sozialer Wohnungsbau errichtet wurde (ARING und HERFERT 2001, S. 49f), der vor allem durch die Gastarbeiterzuwanderung notwendig wurde und die soziale Polarisierung somit schon früh vorangetrieben hat (ARING und HERFERT 2001, S. 51; BraKe et al. 2005, S. 32). Für Walter Prigge bestehen solche Konzentrationsmuster jedenfalls bereits, da sich neben den sozial ghettoisierten, von großstädtischen Problemen bereinigten Lebenswelten der Mittelschichten auch in der Suburbia sozialräumliche Problemfälle entwickeln. Dort konkurrieren Arbeiter aus dem industriellen Sektor mit Angestellten des niedrig qualifizierten Dienstleistungssektors sowie ,insbesondere Migranten um Wohnungen, Lebensweisen und zunehmend um die Dominanz sogenannter ,einheimischer' oder ,fremder‘ Kulturen“ (Prigge 1998, S. 10).

Nachdem nun kurz auf die Postsuburbanisierung im Allgemeinen sowie auf einige alpenspezifische Auswirkungen eingegangen wurde, folgt im nächsten Kapitel die Vorstellung der Untersuchungsregion.

\subsection{Die LEADER-Region Vorderland-Walgau-Bludenz}

LEADER (Abkürzung von Liaison entre actions de développement de l'économie rura$l e$, ,Verbindung zwischen Aktionen zur Entwicklung der ländlichen Wirtschaft“) ist ein Maßnahmenprogramm der Europäischen Union, mit dem seit 1991 modellhaft innovative Aktionen im ländlichen Raum gefördert werden. Lokale Aktionsgruppen erarbeiten vor Ort Entwicklungskonzepte. Ziel ist es, die ländlichen Regionen Europas auf dem Weg zu einer eigenständigen Entwicklung zu unterstützen.

Die LEADER-Region Vorderland-Walgau-Bludenz nimmt in der Förderperiode 20142020 erstmals am LEADER-Programm teil. Die Region selbst umfasst 26 Gemeinden mit etwa 80.000 Einwohnern und lässt sich aus institutioneller Sicht auf die beiden „Regios“ (Regionalentwicklungsgemeinschaften) Vorderland-Feldkirch und Walgau sowie auf die Stadt Bludenz aufteilen (vgl. Abb. 1). Die Stadt Feldkirch, die Mitglied der Regio Vorder- 


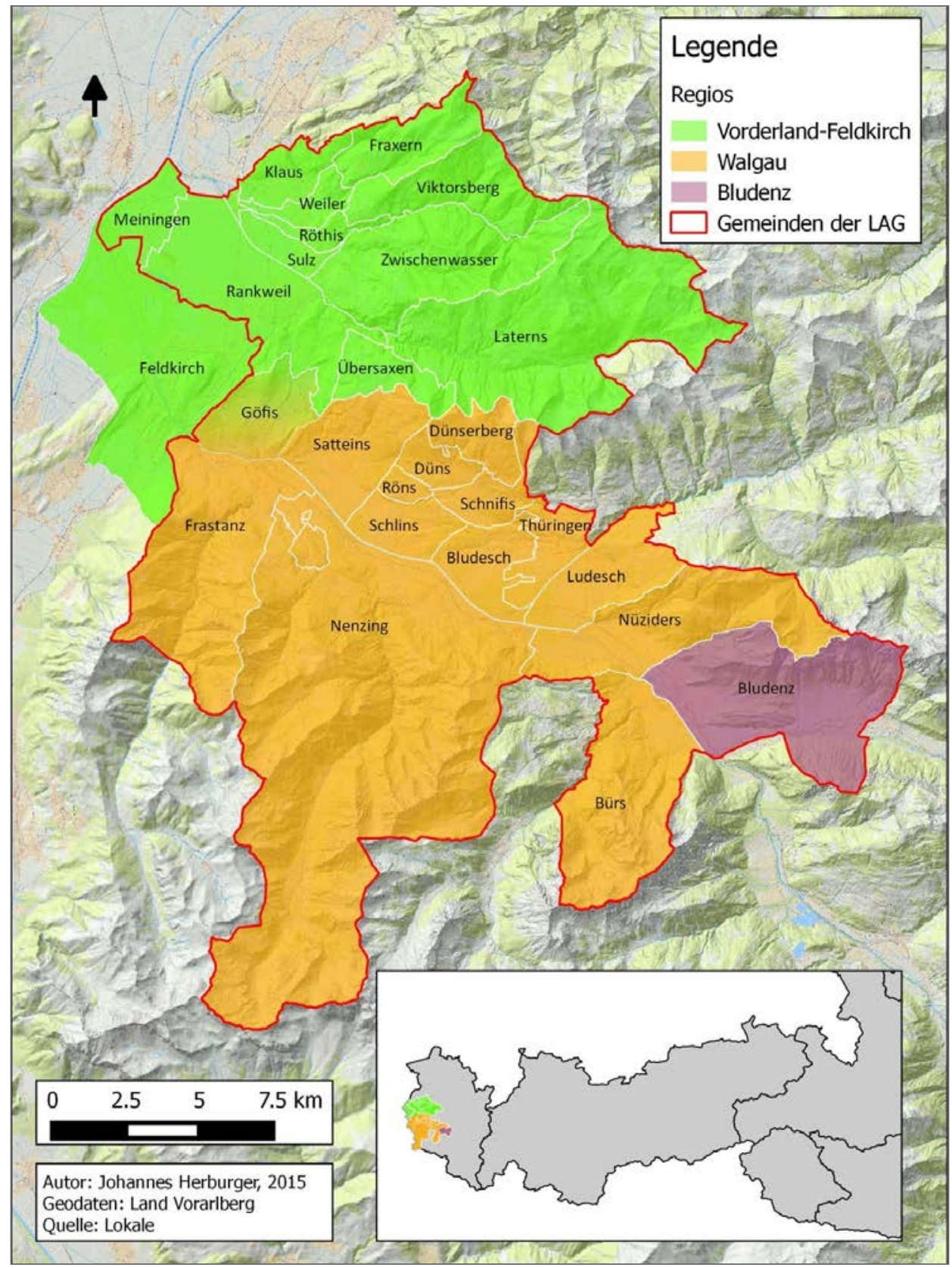

Quelle: Entwurf J. Herburger 2015 auf Basis von Geodaten des Landes Vorarlberg (data.vorarlberg.gv.at) und LAG Vorderland-Walgau-Bludenz (2014)

Abb. 1: Lage und Gemeinden der Untersuchungsregion 
land-Feldkirch ist, ist aufgrund ihrer Größe von über 30.000 Einwohnern nicht über das LEADER-Programm förderberechtigt, wobei eine Förderzusage mit dem Land Vorarlberg besteht, dass bei geeigneten Themen Kooperationen mit der Stadt Feldkirch möglich sind (LAG Vorderland-Walgau-Bludenz 2014, S. 5). Aufgrund dessen sowie angesichts der zentralen Lage und der engen Verflechtungen ist die Stadt Feldkirch in der vorliegenden Untersuchung ebenfalls enthalten und ist in allen Grafiken und Berechnungen inkludiert. Mit der Stadt Feldkirch wächst die Untersuchungsregion auf etwa 112.000 Einwohner an, was etwa 30 Prozent der gesamten Vorarlberger Bevölkerung ausmacht.

Der nördliche Teil der LEADER-Region wird durch die 13 Gemeinden der Regio Vorderland-Feldkirch gebildet, die im südlichen Teil des Vorarlberger Rheintals liegt. Der Walgau, dem insgesamt 14 Gemeinden angehören, beginnt südlich der Stadt Feldkirch und liegt im Einzugsbereich des Flusses Ill. Die Stadt Bludenz bildet das südöstliche Ende der LEADER-Region.

Im Bezug zum theoretischen Ausgangspunkt der Postsuburbanisierung sollte allerdings angemerkt werden, dass sich die klassische „Urbia“, die den Kristallisationskern für die (Post-)Suburbanisierung bildet, nicht in direktem Umfeld der LEADER-Region finden lässt. Diese ist in Bezug auf die Gemeindestrukturen vielmehr durch eine Vielzahl an kleinen Gemeinden, die teilweise auch im Hanggebiet des Rheintals und des Walgaus liegen, mit ursprünglich ländlichen Traditionen geprägt. Diese Gemeindestruktur wurde über die Jahrzehnte hinweg, insbesondere aber seit den 1950er Jahren, durch die fortschreitende Industrialisierung und den Einfamilienhausbau immer mehr überprägt. Ein Blick auf die Bevölkerungsentwicklung verdeutlich die dynamische Entwicklung: Während es in der LEADER-Region zwischen 1951 und 2015 zu einer Verdoppelung der Einwohnerzahlen kam, stieg die österreichische Gesamtbevölkerung im selben Zeitraum um lediglich 26 Prozent, wobei es vor allem im Zeitraum zwischen 1961 und 1971 zu einer starken Öffnung der Schere kam.

Allein in dieser verhältnismäßig kurzen Zeitspanne von zehn Jahren stieg die Bevölkerungszahl der LEADER-Region (inklusive Feldkirch) um knapp 25 Prozent von 64.000 auf 81.000 Einwohner bzw. an. Diese dynamische Entwicklung ist, neben dem damals stattfindenden „Baby-Boom“, auch vor dem Hintergrund der damaligen wirtschaftlichen Entwicklung zu betrachten. Die Vorarlberger Wirtschaft expandierte in dieser Zeit kräftig und allein der industrielle Sektor konnte seine Wertschöpfung verdreifachen. Der industrielle Aufschwung nach dem Zweiten Weltkrieg führte dazu, dass es im Rheintal und den Seitentälern [Anm.: zu denen auch der Walgau zählt] schon Mitte der 1950er Jahre nur mehr geringe Arbeitskräftereserven gab (BURTSCHER 2009, S. 41).

Während in den 1950er Jahren in Vorarlberg noch überwiegend Arbeitskräfte aus Kärnten und der Steiermark angeworben wurden, fand ab den 1960er Jahren eine Internationalisierung der Zuwanderung statt. Gastarbeiter für die damals vorherrschende Textilindustrie wurden primär aus der Türkei und Ex-Jugoslawien angeworben. Dies bewirkte, dass sich zwischen 1961 und 1971 die Zahl der Ausländer in Vorarlberg mehr als verdreifachte (von 7.702 auf 25.534 Personen). Durch Familienzusammenführungen sowie die positive wirtschaftliche Entwicklung erfolgte ein weiterer Anstieg, wodurch Vorarlberg nach Wien zum Bundesland mit dem höchsten Anteil ausländischer Mitbürger in Österreich wurde (BURTSCHER 2009, S. 42f). 
Die wirtschaftliche Expansion und der damit einhergehende Wohlstand führten des Weiteren dazu, dass das Bruttoregionalprodukt (BRP) pro Kopf mit 24.000 öS schon im Jahr des Staatsvertrags 1955 deutlich über dem österreichischen BIP (20.500 öS) lag (Bleyle o. J, S. 17). Das frühe Entstehen von ersten Einfamilienhaussiedlungen war somit eine Konsequenz dieser Entwicklung. Zusammen mit der Zunahme der genannten Immigrationsbewegungen und der Entwicklung des sozialen Mietwohnungsbaus entstanden bauliche Strukturen, die die sozialräumliche Struktur der Region bis heute prägen.

In einer Klassifikation der alpinen Stadtregionen von Perlik, Messerli und Bätzing aus dem Jahr 2001 wird die Untersuchungsregion als industriell und tertiär geprägte Stadtregion bezeichnet, wobei Vorarlberg allgemein eine hochspezialisierte und in die globalen Produktionsnetzwerke integrierte Wirtschaftsstruktur beschieden wird (PERLIK et al. 2001, S. 249). Grundlage dafür war, dass der wirtschaftliche Transformationsprozess von der Textilindustrie hin zur Eisen-, Metall- und Elektronikindustrie sehr gut vollzogen werden konnte. „Der Strukturwandel der Industrie hat sich relativ rasch und unspektakulär vollzogen. Anders als in der ostösterreichischen Grundstoffindustrie gab es keine strukturkonservierenden staats- und landespolitischen Interventionen“ (LUKESCH et al. 2010, S. 58).

Die wirtschaftliche Entwicklung der Untersuchungsregion ist somit vor allem an die Einbettung in die globalen bzw. internationalen Produktions- und Wirtschaftsnetzwerke geknüpft. Die LEADER-Region profitierte und profitiert dabei sicherlich von ihrer zentralen Lage im Viereck München - Stuttgart - Zürich - Mailand, wodurch sich im Lauf der Jahrzehnte ein postsuburban geprägtes Umfeld entwickelte, das sehr stark von der gewerblich-industriellen Wirtschaftsstruktur der Region beeinflusst wird.

Daraus resultiert, dass in einer alpinen Stadtregion, wie etwa der Untersuchungsregion, grundsätzlich auch die gleichen siedlungsstrukturellen Prozesse ablaufen wie außerhalb der Alpen. „Die Alpen spielen daher nicht die Rolle einer Region, in der prinzipiell andere Entwicklungen ablaufen, sondern die Rolle einer Region, in der aufgrund anderer Voraussetzungen die gleichen Entwicklungsprozesse andere Auswirkungen haben“ (PERLIK 2001, S. 172).

\subsection{Zwischenfazit}

Die Ausführungen in den vorherigen Kapiteln haben gezeigt, dass es schwierig ist, von einer spezifischen alpinen Postsuburbia zu sprechen, da es prinzipiell die gleichen Prozesse sind, die zur wirtschaftlichen, baulichen wie auch zur sozialräumlichen Entwicklung eines solchen Siedlungstypus beitragen. Allerdings werden diese Prozesse durch die geographischen und gesellschaftlich-historischen Spezifika des Alpenraums beeinflusst und konfiguriert, wodurch es gerade in den großen Tälern der Alpen zur Herausbildung von bandförmigen Siedlungsstrukturen gekommen ist, die aufgrund postfordistischer Standortmuster mit immer neuen Funktionen aufgefüllt werden und sowohl die Wirtschaftsleistung als auch die Bevölkerung immer mehr in sich konzentrieren.

Allerdings dürfte der wesentliche Einfluss der Alpen mit ihren naturräumlichen und politischen Grenzziehungen auf die Siedlungsentwicklung der sein, dass sich aufgrund 
der einstigen peripheren Lage und des niedrigen Anteils des Dauersiedlungsraums keine Großstädte oder Metropolregionen herausbilden konnten. Diese liegen im perialpinen Bereich und beeinflussen die alpinen Regionen auf unterschiedlichste Art und Weise durch Migrations- und Wirtschaftsnetzwerke. Durch die wirtschaftliche Entwicklung in den Alpen und die in den vorherigen Kapiteln beschriebenen Prozesse dürften sich auch die sozialräumlichen Muster innerhalb der alpinen Stadtregionen ausdifferenziert haben.

Die zentralen Forschungsfragen sind nun, ob sich in der ursprünglich kleinstädtisch-ländlich geprägten Untersuchungsregion dieselben Prozesse abspielen wie in den Randgebieten der außeralpinen Großstädte und ob sich daraus auch entsprechend ausdifferenzierte sozialräumliche Muster entwickelt haben. Weiter operationalisiert wird dies durch die folgenden Fragestellungen:

- Welche besonderen raumordnerischen und sozialräumlichen Problembereiche lassen sich in der Region vorfinden?

- Sind diese Strukturen aufgrund der verhältnismäßig geringen Größe der Kernstadt so ausgeprägt, dass die Kernstadt sozialräumlich kaum mehr vom Umland zu trennen ist?

- Gerade hinsichtlich der Industrialisierung der Alpen in der Nachkriegszeit ist zu ergründen, welchen Einfluss diese auf die heutigen Strukturen ausübt und ob dies auch in kleinstädtischen Regionen die Herausbildung von sozialräumlichen Segregationsmustern beeinflusst.

- Welche zukünftigen Herausforderungen für die sozialräumlichen Strukturen sind durch die Prozesse der De-Agrarisierung, Alterung und Migration zu erwarten?

- Welche Strukturen weisen Gemeinden auf, die zwar funktional mit der Kernstadt verbunden sind, aber aufgrund ihrer Hang- oder Berglage ihren dörflichen Charakter größtenteils behalten konnten?

- Welche Rolle, quantitativ wie auch qualitativ, spielt das Einfamilienhaus als raumprägendes Element unterschiedlichster Generationen?

Um diese Fragen zu beantworten, werden im weiteren Verlauf der Arbeit Indikatoren aus diesen theoretischen Grundlagen abgeleitet, die dann mittels Hauptkomponenten- und Clusteranalyse untersucht werden, um darauf aufbauend zu einer Raumtypisierung zu gelangen.

\section{Forschungsdesign und Methodik}

Die Sozialraumanalyse wurde Ende der 1940er Jahre von Eshref SHEvky und Wendell BELL entwickelt und ging im Gegensatz zu den sozialökologischen Modellen der Chicagoer Schule nicht von der Gesamtstadt aus, sondern befasste sich mit der sozialräumlichen Gliederung der Stadt von „unten“ heraus - sprich von der Stadtteilebene aus. Es wird davon ausgegangen, „dass die Gesamtstadt die Summe vieler kleiner, in sich abgeschlossener ,Welten“ darstellt“, welche als „Natural Areas“ tituliert werden (FASSMANN 2009, S. 134). Die „social area analysis“" stellt daher eine operationale Forschungstechnik dar, um die sozialräumliche Differenzierung von Großstädten vor dem Hintergrund der sich verändernden gesellschaftlichen Strukturen zu untersuchen (B̈̈HR 2010, S. 124). 


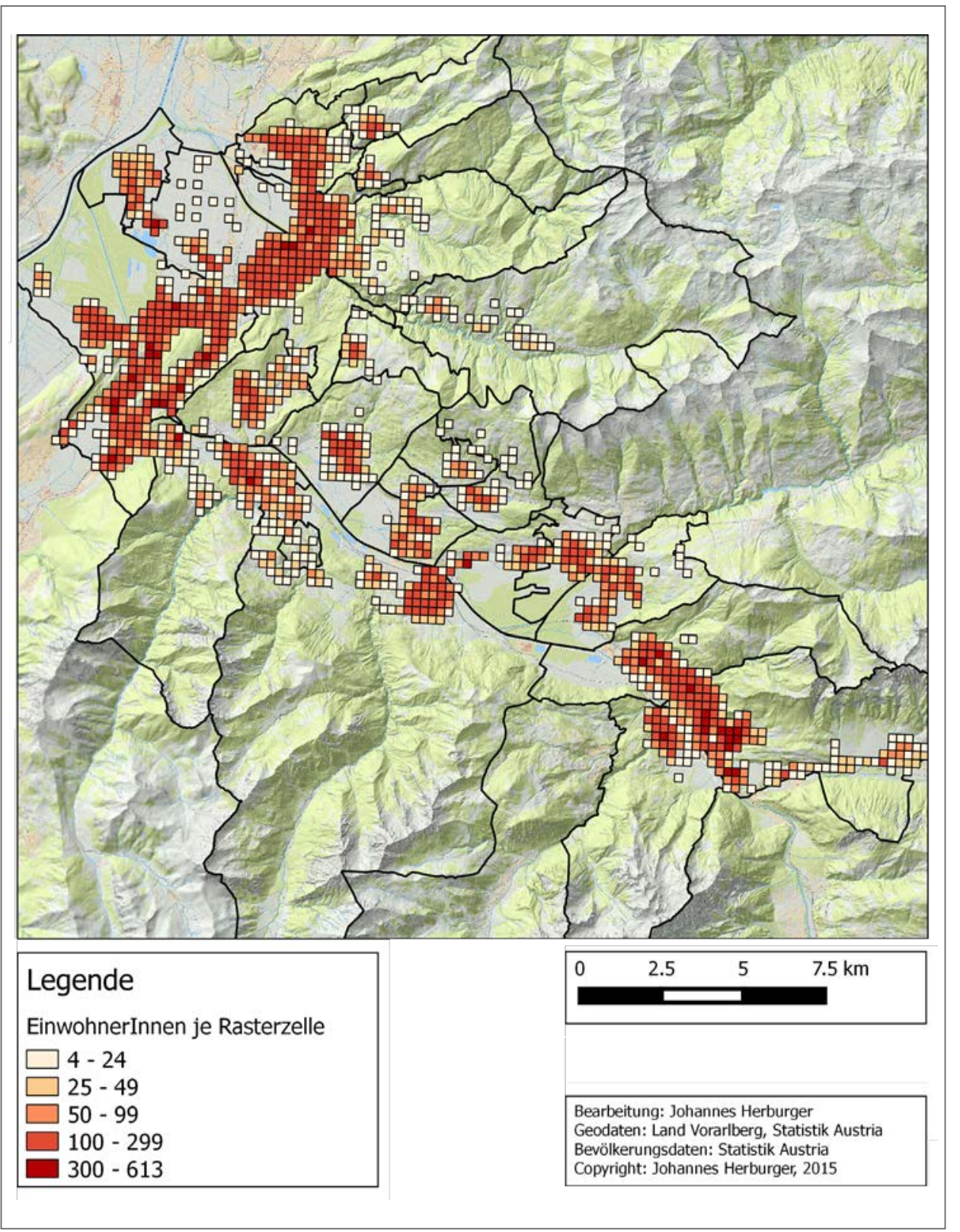

Quelle: Eigener Entwurf J. Herburger 2015 auf Basis von Daten der Bundesanstalt Statistik Austria und von Geodaten des Landes Vorarlberg (data.vorarlberg.gv.at)

Abb. 2: Bevölkerungsdichte 2011 in der LEADER-Region Vorderland-Walgau-Bludenz auf Rasterdatenebene (250m x 250m Raster) 
Diese - ursprünglich für Großstädte entwickelte - Methodik wird in der vorliegenden Studie auf eine nicht-städtische bzw. postsuburbane Untersuchungsregion übertragen. Dabei kann es zu Komplikationen hinsichtlich der Datenverfügbarkeit kommen. Die „Natural Areas“ als sozial homogene Stadtteile, deren Grenzen natürliche oder künstliche Barrieren darstellen (Flussläufe, Eisenbahnlinien, Straßen) und die mit den statistischen Grenzen übereinstimmen (FASSMANN 2009, S. 134), existieren in dieser Form in der Postsuburbia nicht direkt. Um solche kleinräumigen sozialräumlichen Muster in der Untersuchungsregion zu erkennen, muss auf andere administrative bzw. statistische Einheiten zurückgegriffen werden können.

In dieser Arbeit werden die regionalstatistischen Rasterdatensätze in der Größe 250 x 250 m der Bundesanstalt Statistik Austria verwendet, durch die auch kleinräumige Muster erkannt werden können. Die eben vorgestellte Rastergröße ist auch die kleinste statistische Einheit, auf der Merkmalsausprägungen zu den Fallzahlen verfügbar sind. Im Vergleich zu den „Natural Areas“ von SHEvky und BELL sind diese Einheiten zwar nicht durch linienhafte Infrastrukturen oder Flussläufe begrenzt, allerdings eignen sich diese Daten aufgrund der disperseren Siedlungsstruktur der Postsuburbia dennoch für die Analyse einer solchen Fragestellung.

Alle verwendeten Daten entstammen der Registerzählung des Jahres 2011, die in Gesamt-Österreich anstelle der bisher üblichen „klassischen“ Volkszählungen durchgeführt wurde. Einzige Ausnahme ist der Bevölkerungsstand der Volkszählung 2001, der für die Berechnung der Bevölkerungsentwicklung als dynamischer Indikator notwendig war. Die Volkszählung aus dem Jahr 2001 ist auch der älteste Datenbestand, der bei der Statistik Austria für die regionalstatistischen Rastereinheiten verfügbar ist.

Es konnten nur jene Rasterzellen in die Analyse einfließen, die eine Fallzahl an Personen, Gebäuden, Familien und Haushalten von mindestens 1 (bzw. bei Personen 4) aufweisen, wodurch insgesamt 1.309 Rasterzellen mit einer Fläche von 81,81 km² und 110.045 Einwohnern untersucht werden. Einige Zellen konnten wegen ihrer zu geringen Einwohnerzahl nicht in die Analyse aufgenommen werden konnten, was durch die eben genannten Restriktionen bedingt ist. Anhand der Karte zur Bevölkerungsdichte wird ersichtlich, wie sich die Rasterzellen über die Region verteilen (Abb. 2). Deutlich erkennbar ist die weitgehend zusammenhängende Siedlungsstruktur.

\section{Der Weg zur Raumtypisierung - Indikatoren und Analyse}

Die Berechnung und Verwendung von einfachen Anteilswerten als Indikatoren hat den Vorteil, dass sich höhere Korrelationen ergeben, wie sich während des Analyseprozesses herausstellte. Hohe Korrelationen sind schließlich auch die Grundvoraussetzung für die nachfolgenden multivariaten Analysen.

An dieser Stelle muss noch angemerkt werden, dass Metaprozesse wie die Industrialisierung, die über Jahrzehnte zurückreichen, aufgrund der Datenverfügbarkeit auf dieser Ebene also kaum dynamisch darzustellen sind. Die Analyse des sozialräumlichen Status Quo auf dieser kleinteiligen Bezugsebene erlaubt es aber, die Auswirkungen, die diese Prozesse mit sich bringen, im Detail zu untersuchen. 
Die ausgewählten Indikatoren sind in der folgenden Tabelle übersichtsmäßig dargestellt, wobei auch angemerkt ist, welche unterschiedlichen Dimensionen die Indikatoren messen sollen (Tab. 1).

\begin{tabular}{|c|c|}
\hline Indikator & Dimension der Indikatoren \\
\hline Bevölkerungsentwicklung 2001-2011 & Bevölkerungsentwicklung \\
\hline Anteil der über 65-jährigen Wohnbevölkerung & \multirow{2}{*}{ Demographischer Wandel } \\
\hline Anteil der Bewohner mit Geburtsland Türkei oder Ex-Jugoslawien & \\
\hline Anteil an Pflichtschulabsolventen & \multirow{2}{*}{ Bildung } \\
\hline Akademikerquote & \\
\hline Bevölkerungsdichte & \multirow{2}{*}{$\begin{array}{l}\text { Bauliche und } \\
\text { Bevölkerungs-Dichte }\end{array}$} \\
\hline Gebäudedichte & \\
\hline Anteil an Ein- und Zwei-Personen-Haushalten & \multirow{3}{*}{$\begin{array}{l}\text { Familien- und Haushalts- } \\
\text { struktur }\end{array}$} \\
\hline Anteil an Ein-Eltern-Familien & \\
\hline Anteil an Ehepaar-Kind-Familien & \\
\hline Anteil der Gebäude mit drei oder mehr Geschossen & \multirow{3}{*}{$\begin{array}{l}\text { Gebäudestrukturelle } \\
\text { Merkmale }\end{array}$} \\
\hline Anteil an Einfamilienhäusern & \\
\hline Anteil der Gebäude aus der Periode 1945-1970 & \\
\hline
\end{tabular}

Quelle: Eigene Zusammenstellung. Datengrundlage: Daten der Registerzählung 2011 und der Volkszählung 2001

Tab. 1: Ausgewählte Indikatoren für die Raumtypisierung

Die in dieser Arbeit verwendeten statistischen Methoden lassen sich den multivariaten statistischen Analyseverfahren zuordnen. Die Faktoren- bzw. Hauptkomponentenanalyse sowie die Clusteranalyse gehören zur Gruppe der strukturentdeckenden multivariaten Verfahren, deren Nutzen vor allem durch die datenreduzierende Vorgehensweise sehr groß ist. „Diese Verfahren stellen ein Instrumentarium zur Verfügung, mit dem [...] Variablen simultan bearbeitet werden können. Sie tragen damit der Tatsache Rechnung, dass sich in der Regel verschiedene Variablen [...] wechselseitig beeinflussen“ (BüLOw 1996, S. 1).

\section{Faktoren- bzw. Hauptkomponentenanalyse}

Die Korrelationen der Variablen untereinander sind als Ausgangspunkt für die Hauptkomponentenanalyse zu betrachten, da so die wechselseitigen Beziehungen der Variablen dargestellt werden können. In weiterer Folge wird der Datensatz durch die Datenreduktion nicht nur vereinfacht, sondern es werden neue Variablen bzw. Komponenten geschaffen, die die strukturellen Beziehungen der Variablen unter möglichst geringem Informationsverlust zusammenfassen (ZöFEL 2002, S. 182).

Im Softwarepaket SPSS können zwei weitere Verfahren zur Beurteilung der Eignung eines Datensatzes verwendet werden (BüLow 1996, S. 9). Zum einen prüft der Bart- 
lett-Test, ob die Zusammenhänge in der Korrelationsmatrix zufällige Schwankungen um den wahren Wert Null sind. Ein hohes ungefähres Chi-Quadrat von 5.034,051 und ein niedriges Signifikanz-Niveau bedeuten, dass die Zusammenhänge in der Korrelationsmatrix keine zufälligen Schwankungen um einen wahren Wert von Null sind (BüLow 1996, S. 9). Das Kaiser-Mayer-Olkin-Maß für die Angemessenheit der Stichprobe sollte nahe 1 liegen (BüLow 1996, S. 11) wobei ein Wert von 0,632, wie in unserem Modell, eine mittlere Eignung für eine Hauptkomponentenanalyse bedeutet (NoRUsis 1993, S. 53f).

Da die Aufgabe der Hauptkomponentenanalyse aber die Reduzierung der Komplexität des Datenbestandes ist, also eine Reduktion der Variablenanzahl (ZöFEL 2002, S. 182), müssen nun die Eigenwerte beobachtet werden. Diese stellen den Anteil der erklärten Varianz einer Komponente dar. Um mehr Sicherheit mit der Bestimmung der richtigen Anzahl an Komponenten zu bekommen, gibt es Hilfsmittel, wie das Kaiser-Guttmann-Kriterium, welches besagt, dass nur Komponenten in das Modell einbezogen werden sollen, die einen Eigenwert $>1$ besitzen (BACKHAus et al. 1990, S. 90). Die vier ersten Komponenten weisen einen Eigenwert $>1$ auf und erklären bereits mehr als die Hälfte (59,9 \%) der gesamten Varianz aller Variablen (Tab. 2).

\begin{tabular}{|c|c|c|c|c|c|c|}
\hline \multirow{2}{*}{$\begin{array}{c}\text { Kompo- } \\
\text { nente }\end{array}$} & \multicolumn{4}{|c|}{ Anfängliche Eigenwerte } & \multicolumn{3}{|c|}{$\begin{array}{c}\text { Erklärte Gesamtvarianz } \\
\text { Sumen von quadrierten Faktor- } \\
\text { ladungen für Extraktion }\end{array}$} \\
\cline { 2 - 7 } & Gesamt & $\begin{array}{c}\text { \% der } \\
\text { Varianz }\end{array}$ & $\begin{array}{c}\text { Kumu- } \\
\text { lierte \% }\end{array}$ & Gesamt & $\begin{array}{c}\text { \% der } \\
\text { Varianz }\end{array}$ & $\begin{array}{c}\text { Kumu- } \\
\text { lierte \% }\end{array}$ \\
\hline 1 & 2,912 & 22,397 & 22,397 & 2,912 & 22,397 & 22,397 \\
\hline 2 & 2,133 & 16,408 & 38,805 & 2,133 & 16,408 & 38,805 \\
\hline 3 & 1,467 & 11,285 & 50,090 & 1,467 & 11,285 & 50,090 \\
\hline 4 & 1,274 & 9,802 & 59,892 & 1,274 & 9,802 & 59,892 \\
\hline 5 &, 950 & 7,310 & 67,202 & & & \\
\hline \multicolumn{7}{|c|}{ Zugunsten der Übersichtlichkeit wurden einige Zeilen gelöscht. } \\
\hline 13 & \multicolumn{7}{|c|}{, 108} & \multicolumn{7}{|c|}{, 827} & 100,000 & & & \\
\hline
\end{tabular}

Quelle: Eigene Berechnung unter Verwendung des Programmsystems SPSS. Datengrundlage: Daten der Registerzählung 2011 und der Volkszählung 2001

Tab. 2: Ergebnis der Hauptkomponentenanalyse

Um die Interpretierbarkeit der Faktorlösung zu erhöhen, wird in der Regel die Faktorladungsmatrix verändert (vgl. BüLow 1996, S. 17). Das Ziel eines solchen Vorgehens ist es, die Komponenten so deutlich wie möglich darzustellen. Die Ladungen von Variablen sollen somit auf die stärker korrelierende Komponente erhöht werden, um die Variablenstruktur, die eine Komponente konstituiert, klarer zu machen. Durch Rotation des Achsenkreuzes, auf dem die Variablen liegen, kann die Zuordnung einer Variablen zu einer Komponente erleichtert werden. BüLOw (1996, S. 17) und ZöFEL (2002, S. 153) schlagen hierfür die sogenannte Varimax-Methode vor. Dabei wird das Achsenkreuz rechtwinklig 
rotiert, sodass die Varianz der Ladungen je Komponente maximiert wird und auch die Unabhängigkeitsprämisse der Komponenten beibehalten werden kann (BACKHAUs et al. 1990: S. 92). Durch die Varimax-Rotation laden die Variablen nun eindeutig auf eine der vier Komponenten, womit die Zuordnung der Variablen zu einer Komponente und damit das gesamte Modell erheblich vereinfacht werden (BACKHAus et al. 1990, S. 92).

\section{Kategorisierung der Rasterzellen - die Clusteranalyse}

Mit Hilfe der in der Hauptkomponentenanalyse errechneten vier Komponenten kann nun die Clusteranalyse durchgeführt werden, wenngleich mit der Festlegung auf ein Verfahren das Resultat des Clusterprozesses gleichsam vorgegeben wird. Daher stützt sich die Auswahl der Verfahren auf Empfehlungen aus der Literatur, in der grundsätzlich dazu geraten wird, mittels der Ward-Methode die Anzahl der Cluster zu fixieren, um danach die Zuteilung der Rasterzellen zu den Clustern durch die k-Means-Methode zu verbessern (BüLow 1996, S. 32).

Die Klassifizierung von Objekten (in diesem Fall von Rasterzellen) mittels Clusteranalyse ist ein induktives Verfahren, „indem sie Klumpen (= Cluster) von ähnlichen Raumeinheiten aufspürt, wobei die Ähnlichkeit durch die Lage der Raumeinheiten in dem von den Variablen [Anm.: bzw. Komponenten] gebildeten Koordinatensystem bestimmt wird“ (BAhrenberg et al. 1992, S. 279). Der Ablauf einer Clusteranalyse gliedert sich in zwei grundlegende Schritte (BACKHAUs et al. 1990, S. 116):

Erster Schritt: Wahl des Proximitäts- bzw. Distanzmaßes: Messung der Unterschiede zwischen den Objekten mittels unterschiedlicher Berechnungsmethoden. Die berechnete Zahl symbolisiert die Ähnlichkeit der Objekte. Für diese Analyse wurde das euklidische Distanzmaß verwendet, welches im Groben der „Luftlinie“ zwischen den Rasterzellen im vierdimensionalen Koordinatensystem entspricht. Da andere Maße, wie etwa der Korrelationskoeffizient, stark von der Größe der Raumeinheiten abhängen können, kann das euklidische Distanzmaß als praktikabel angesehen werden, da alle Rasterzellen die gleiche Größe aufweisen.

Zweiter Schritt: Wahl des Fusionierungsalgorithmus: Mittels der durch das Distanzmaß errechneten Werte werden die Objekte so gruppiert, dass sie sich möglichst ähnlich sind. Dazu wurde zuerst mit dem Ward-Verfahren eine erste Zuordnung hergestellt, um diese in einem zweiten Schritt durch das k-means-Verfahren zu verfeinern.

Ein weiterer heikler Schritt ist die Bestimmung der richtigen Clusteranzahl, denn mit jedem Fusionierungsschritt wächst der Grad der Generalisierung und damit auch der Verlust von Informationen. Neben dem induktiven Vorgehen des Experimentierens kann man sich auch an sprunghaften Anstiegen der Heterogenität bzw. der Fehlerquadratsumme orientieren (BüLow 1996, S. 33). Ein Schritt, bei dem eine stärkere Zunahme der Fehlerquadratsumme beobachtet werden kann, ist Schritt 1302, bei dem die Fehlerquadratsumme um 48 steigt, während es davor nur Werte um 30 waren. Auch danach kommt es zu einem kontinuierlichen Anstieg dieses Wertes, weshalb die optimale Clusteranzahl mit sieben Clustern festgelegt wurde. Es wurden deshalb sieben Cluster gebildet, weil die Fallzahl der Rasterzellen 1309 beträgt und der Fusionierungsalgorithmus nach dem 1302. Schritt abgebrochen wurde: 1309 - 1302 = 7 (siehe dazu BAHrENBERG et al. 1992, S. 293). 


\section{Sozialräumliche Typisierung der LEADER-Region Vorderland-Walgau-Bludenz}

In Tabelle 3 sind die durch die Clusteranalyse ermittelten sieben Cluster (= Raumtypen) im Überblick einschließlich einer Kurzbeschreibung aufgelistet, während in Tabelle 4 die Werte der Indikatoren je Raumtyp angeführt werden. Die Ergebnisse verstehen sich dabei als Mittelwerte über alle Rasterzellen des jeweiligen Raumtyps hinweg. In Abbildung 3 wird schließlich die räumliche Ausprägung und Verteilung der Raumtypen dargestellt. Zur Bewertung der Raumtypen werden die folgenden formellen und informellen Instrumente herangezogen: Neben dem Vorarlberger Raumplanungsgesetz und der Lokalen Entwicklungsstrategie (LES) dienen die „Vision Rheintal“, das „Räumliche Entwicklungskonzept (REK) Walgau“ sowie das „Vorarlberger Integrationsleitbild“ als Bewertungsgrundlage.

\begin{tabular}{|l|c|l|}
\hline \multicolumn{1}{|c|}{ Raumtyp } & $\begin{array}{l}\text { Anzahl } \\
\text { Raster- } \\
\text { zellen }\end{array}$ & \multicolumn{1}{|c|}{ Beschreibung } \\
\hline $\begin{array}{l}\text { Hochverdichtete } \\
\text { Zentren }\end{array}$ & 118 & $\begin{array}{l}\text { Orts- und Stadtzentren mit kleinen Haushaltsgrößen und sozial } \\
\text { gemischter Bevölkerung sowie hochverdichtete Bereiche in } \\
\text { nicht-integrierten Lagen }\end{array}$ \\
\hline Postsuburbia & 323 & $\begin{array}{l}\text { Übergangsbereich zwischen Zentrum und Suburbia. Überprägung } \\
\text { historischer Siedlungsbereiche durch Einfamilienhaus- sowie ver- } \\
\text { dichteten Wohnungsbau. }\end{array}$ \\
\hline $\begin{array}{l}\text { Ältere Suburbani- } \\
\text { sierungsbereiche }\end{array}$ & 331 & $\begin{array}{l}\text { Siedlungsbereiche, die vom Einfamilienhausbau bzw. von den ers- } \\
\text { ten Suburbanisierungswellen geprägt sind. Aufgrund der Alterung } \\
\text { unterdurchschnittlicher Anteil an Ehepaar-Kind-Familien. }\end{array}$ \\
\hline $\begin{array}{l}\text { Jüngere Suburba- } \\
\text { nisierungsbereiche }\end{array}$ & 171 & $\begin{array}{l}\text { Insbesondere Siedlungsrandbereiche, die durch starkes Bevöl- } \\
\text { kerungswachstum gekennzeichnet sind. Klassisch suburbane } \\
\text { Familien- und Gebäudestruktur. }\end{array}$ \\
\hline $\begin{array}{l}\text { Bereiche mit } \\
\text { starker sozialer } \\
\text { und ethnischer } \\
\text { Segregation }\end{array}$ & 91 & $\begin{array}{l}\text { Bereiche mit überdurchschnittlicher Konzentration von Personen } \\
\text { mit Geburtsland Türkei oder Ex-Jugoslawien. Hoher Anteil an } \\
\text { Personen mit niedrigem Bildungsstand. Lage oft an den Sied- } \\
\text { lungsrandbereichen bzw. in der Nähe ehemaliger Textilindustrie- } \\
\text { betriebe. }\end{array}$ \\
\hline $\begin{array}{l}\text { Von Überalterung } \\
\text { geprägte Sied- } \\
\text { lungsbereiche }\end{array}$ & 125 & $\begin{array}{l}\text { Siedlungsbereiche mit Bevölkerungsverlusten und überdurch- } \\
\text { schnittlich hohem Anteil an über 65-Jährigen. Ebenfalls über- } \\
\text { durchschnittlich hoher Anteil an Wohngebäuden aus der Baupe- } \\
\text { riode 1945-1970. }\end{array}$ \\
\hline $\begin{array}{l}\text { Ländlich-Land- } \\
\text { wirtschaftliche } \\
\text { Bereiche }\end{array}$ & 150 & $\begin{array}{l}\text { Sehr dünn besiedelte, meist landwirtschaftliche Bereiche in den } \\
\text { Berggemeinden, auf Riedflächen und in den Siedlungsrandberei- } \\
\text { chen. }\end{array}$ \\
\hline
\end{tabular}

Quelle: J. HERBURGER, eigene Berechnung und Zusammenstellung

Tab. 3: Ergebnis der Clusteranalyse: Kurzvorstellung der sieben Raumtypen im Untersuchungsgebiet 


\begin{tabular}{|c|c|c|c|c|c|c|c|c|c|c|c|c|c|}
\hline Raumtyp & 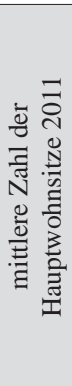 & 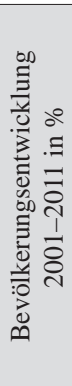 & 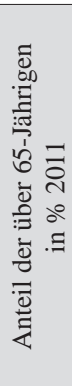 & 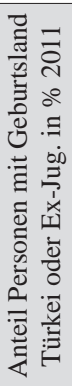 & 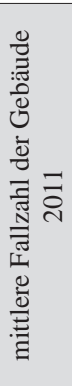 & 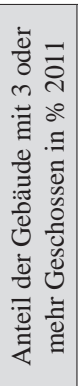 & 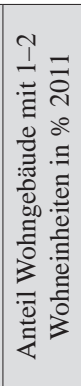 & 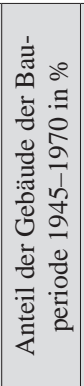 & 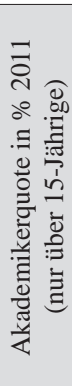 & 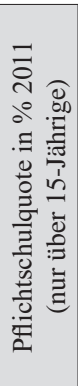 & 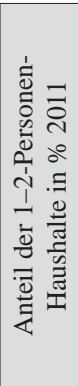 & 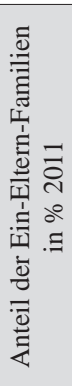 & 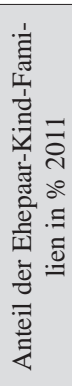 \\
\hline $\begin{array}{l}\text { Hochverdichtete } \\
\text { Zentren }\end{array}$ & 245 & 1,3 & 16,7 & 16,5 & 43 & 42,0 & 38,2 & 22,5 & 9,9 & 36,2 & 70,1 & 18,6 & 35,7 \\
\hline Postsuburbia & 131 & 8,9 & 15,5 & 3,6 & 37 & 14,8 & 68,1 & 23,1 & 12,1 & 26,4 & 60,7 & 13,9 & 2,3 \\
\hline $\begin{array}{l}\text { Ältere Sub- } \\
\text { urbanisierungs- } \\
\text { bereiche }\end{array}$ & 54 & 2,0 & 15,6 & 0,1 & 17 & 8,2 & 72,0 & 22,7 & 6,4 & 32,7 & 54,9 & 12,7 & 46,8 \\
\hline $\begin{array}{l}\text { Jüngere Sub- } \\
\text { urbanisierungs- } \\
\text { bereiche }\end{array}$ & 50 & 34,9 & 7,0 & 0,0 & 15 & 5,3 & 81,4 & 8,4 & 11,6 & 22,4 & 43,4 & 9,6 & 56,2 \\
\hline $\begin{array}{l}\text { Bereiche mit } \\
\text { starker sozialer } \\
\text { und ethnischer } \\
\text { Segregation }\end{array}$ & 87 & 1,9 & 9,4 & 37,1 & 14 & 27,3 & 44,7 & 25,6 & 3,4 & 52,3 & 54,2 & 15,0 & 48,6 \\
\hline $\begin{array}{l}\text { Von Überalterung } \\
\text { geprägte Sied- } \\
\text { lungsbereiche }\end{array}$ & 34 & 12,0 & 31,3 & 0,1 & 11 & 10,6 & 59,7 & 24,2 & 5,0 & 36,6 & 66,2 & 12,2 & 31,9 \\
\hline $\begin{array}{l}\text { Ländlich-Land- } \\
\text { wirtschaftliche } \\
\text { Bereiche }\end{array}$ & 7 & 5,7 & 9,3 & 0,0 & 2 & - & - & - & 6,8 & 24,3 & - & - & - \\
\hline $\begin{array}{l}\text { Untersuchungs- } \\
\text { region } \\
\text { insgesamt }\end{array}$ & 84 & 5,0 & 15,1 & 11,4 & 22 & 16,6 & 63,7 & 22,3 & 9,5 & 31,7 & 62,3 & 13,7 & 42,5 \\
\hline
\end{tabular}

Quelle: J. HerburGer, eigene Berechnung und Zusammenstellung

Tab. 4: Mittelwerte der Indikatoren je Raumtyp

Die räumliche Verteilung der Raumtypen gibt einige klar erkennbare Muster wieder. Charakteristisch ist, dass insbesondere in den größeren Gemeinden außerhalb der dicht besiedelten und bebauten Orts- bzw. Stadtzentren eine Art postsuburbaner Ring entstanden ist. Dies ist sehr deutlich im Vorderland erkennbar, wo gleichsam schon von einem postsuburbanen Siedlungsband, das sich von Feldkirch bis Klaus zieht, gesprochen werden kann. Im Walgau ist dies etwas differenzierter zu betrachten. Hier findet sich dieses Siedlungsband nur zwischen Bludenz und den Nachbargemeinden Nüziders und Bürs sowie in geringerem Ausmaß zwischen Bludesch, Thüringen und Ludesch bzw. Frastanz und Nenzing. Der Walgau ist noch stärker durch klarere Siedlungsgrenzen zwischen den Gemeinden geprägt, wobei die zentralen bzw. postsuburbanen Rasterzellen dem Verlauf der Landesstraße folgen. 


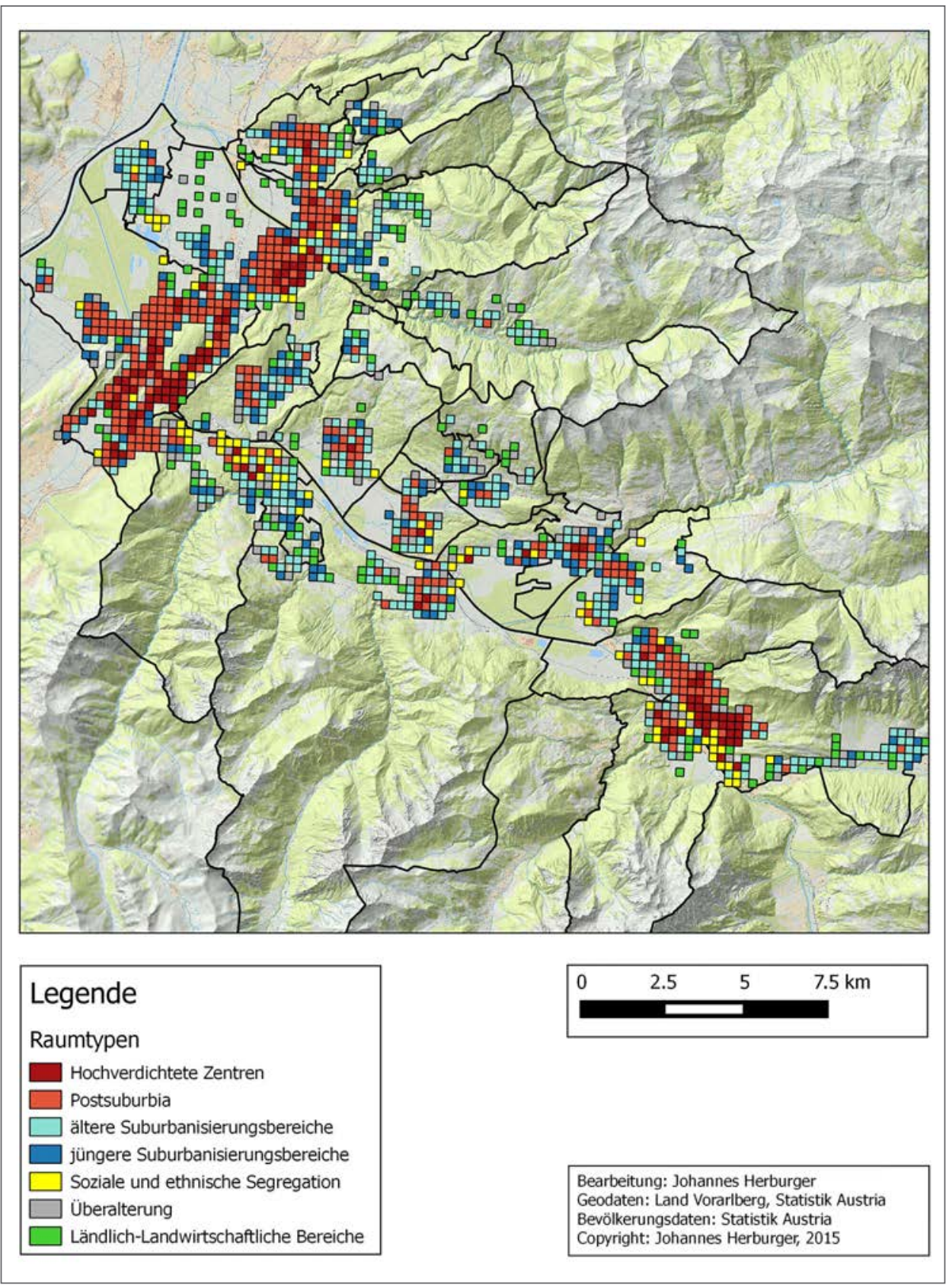

Quelle: J. Herburger, eigener Entwurf

Abb. 3: Sozialräumliche Typisierung der LEADER-Region Vorderland-Walgau-Bludenz 
Grundsätzlich spiegelt die Verteilung der postsuburbanen Rasterzellen die siedlungsstrukturelle Weiterentwicklung der ersten „suburbanen Siedlungen“ entlang der alten Hauptstraßen wider. Dies zeigt sich durch eine sehr heterogene Baustruktur von ehemals landwirtschaftlich genutzten Häusern (deren ungenutzter Wirtschaftsteil oft immer noch besteht), dicht bebauten Einfamilienhaussiedlungen und verdichteten Geschosswohnbauten unterschiedlicher Generationen (siehe Abb. 4). Aufgrund der Lage und Dichte ist die Verkehrsanbindung überdurchschnittlich gut und anders als in den dicht bebauten Ortsund Stadtzentren gibt es noch einiges an Bauflächenpotenzial. Der Veränderungsdruck in diesem Raumtyp zeigt sich in Abbildung 5.

Neben der schwierigen Mobilisierung dieser Bauflächen müssen in Bezug auf diesen Raumtyp noch weitere Rahmenbedingungen berücksichtigt werden. Aufgrund der beschriebenen Eigenschaften kommt es auch zu einer Häufung der Anforderungen, denen dieser Raumtyp Genüge tun sollte und die, angefangen von der Nachverdichtung über die soziale und funktionale Durchmischung bis zu Wohnen im Alter und ähnlichen Themen reichen. Darin liegt einiges an Konfliktpotenzial begraben, da diese Nutzungsansprüche im Widerspruch zur immer noch sehr stark verankerten Einfamilienhausmentalität stehen, wenngleich durch den hohen Immobilienpreisdruck langsam ein Umdenken erkennbar ist.

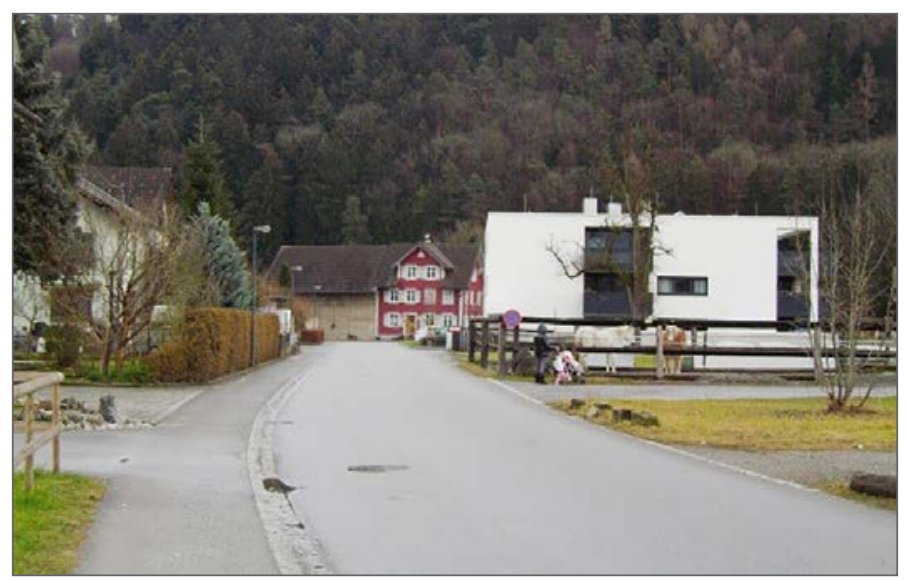

Foto: J. Herburger, 2015

Abb. 4: Charakteristisches Rheintalhaus im Hintergrund und Neubau im Vordergrund

Kennzeichnend für beide Regionen, das Vorderland und den Walgau, ist der Druck auf die Siedlungsränder, der durch die dunkelblauen Rasterzellen in Abbildung 3 dargestellt wird. Diese sind durch starkes Bevölkerungswachstum von 34,9 Prozent im Zeitraum von 2001 bis 2011 und Einfamilienhausbau geprägt und entsprechen größtenteils dem in der Theorie beschrieben familiären Idealbild der Ehepaar-Kind-Familie. Daneben finden sich suburbane Gebiete älterer Generationen, die mittlerweile von kleineren Haushaltsgrößen geprägt sind (knapp 55 Prozent 1- und 2-Personen-Haushalte), da die Kindergeneration aus den Elternhäusern ausgezogen ist. In der folgenden Darstellung (Abb. 6) sind sozusagen idealtypische 


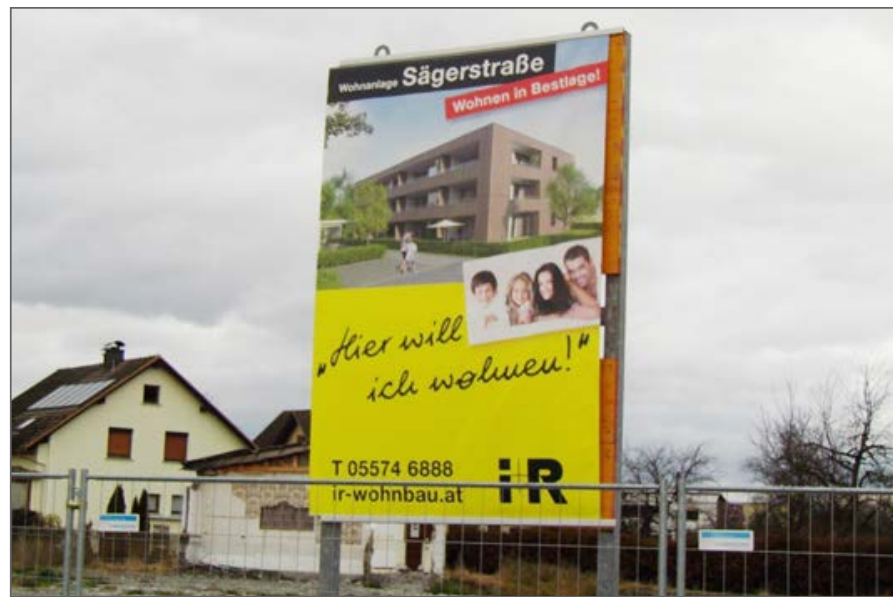

Foto: J. HERBURGER, 2015

Abb. 5: Ankündigung eines modernen Neubaus neben einem Einfamilienhaus der 1960er Jahre

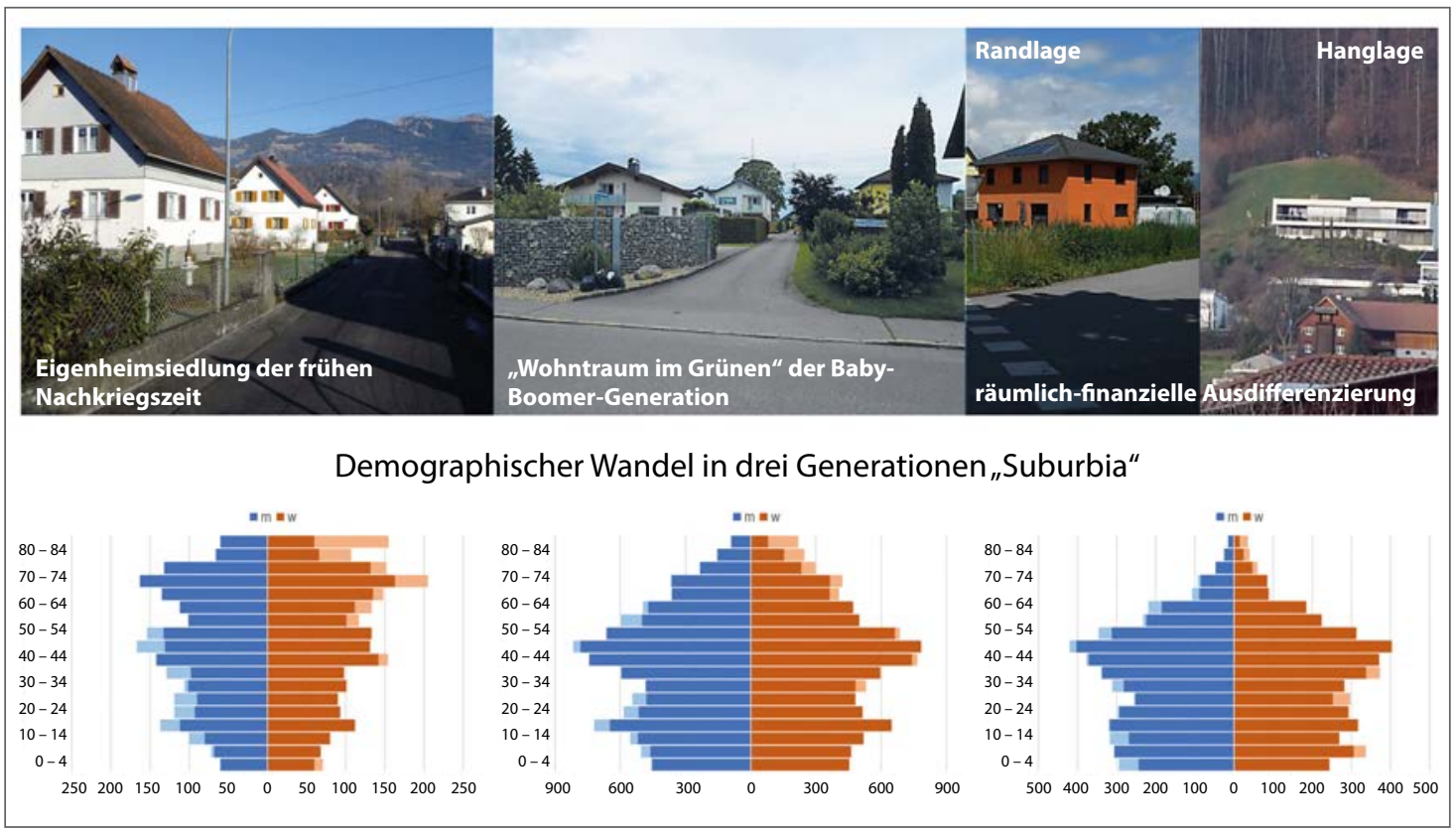

Quellen: J. Herburger, 2015 (Fotos); Altersaufbaudiagramme: Eigener Entwurf, Berechnung auf Basis der Daten der Bundesanstalt Statistik Austria über die Altersstruktur 2011

Abb. 6: Demographischer Strukturwandel - Vergleich des Altersaufbaus der Bevölkerung in drei Raumtypen aus verschiedenen „Generationen“ 
Einfamilienhaussiedlungen und die Bevölkerungsstruktur der Raumtypen „Postsuburbia“, „ältere Suburbanisierungsbereiche“ und ,jüngere Suburbanisierungsbereiche“ dargestellt.

Die unterschiedlichen raum- und sozialplanerischen Problemlagen werden dadurch verdeutlicht. Während im Raumtyp „Postsuburbia“ die Konsolidierung und Nachbesetzung dieser Siedlungen mit jüngerer Bevölkerung im Vordergrund stehen sollte, zeigt der Raumtyp ,junge Suburbanisierungsbereiche“ neben dem dynamischen Wachstum des Siedlungsrandes auch eine deutliche räumlich-finanzielle Ausdifferenzierung auf. Während viele junge Familien sich den Traum vom Eigenheim am Siedlungsrand in Fertighausbauweise verwirklichen, kommt es zur Segregation finanziell besser situierter Bevölkerungsgruppen in den attraktiven Hanglagen der Region. Dazwischen liegt der Raumtyp ,ältere Suburbanisierungsbereiche“, bei dem die Kindergeneration der Baby-Boomer (die im Jahr 2011 10-19-Jährigen) deutlich aus der durchschnittlichen Altersstruktur heraussticht. Da diese Personen mittlerweile selbst auf dem Immobilienmarkt aktiv sein dürften, kommt es also schon in den Einfamilienhausgebieten der 1990er Jahre zu Unternutzungserscheinungen.

Neben diesen Raumtypen, die fast musterhaft einen Dichtegradienten vom Zentrum in die offene Landschaft darstellen, sind die Raumtypen „Von Überalterung geprägte Siedlungsbereiche“ und „soziale und ethnische Segregation“ über die Region verstreut. Dies dürfte primär mit historischen Einzelursachen zusammenhängen. Beim Raumtyp „Von Überalterung geprägte Siedlungsbereiche“ muss beachtet werden, dass dieser auch Rasterzellen enthält, in denen Pflegeheime situiert sind.

Die Ansiedlung des sozialen Mietwohnungsbaus in der Nähe großer Industriebetriebe dürfte für die Herausbildung des Raumtyps „,soziale und ethnische Segregation“ eine wichtige Rolle gespielt haben (siehe Abb. 7). Diesbezüglich zeigen sich klare Konzentrations- bzw. Segregationstendenzen, da in den 91 Rasterzellen (7 \% der gesamten Rasterzellen in der Region), die diesen Raumtyp ausmachen, 25 Prozent aller Personen mit Geburtsland Türkei oder Ex-Jugoslawien leben. Ebenso liegt der Anteil der Pflichtschul-

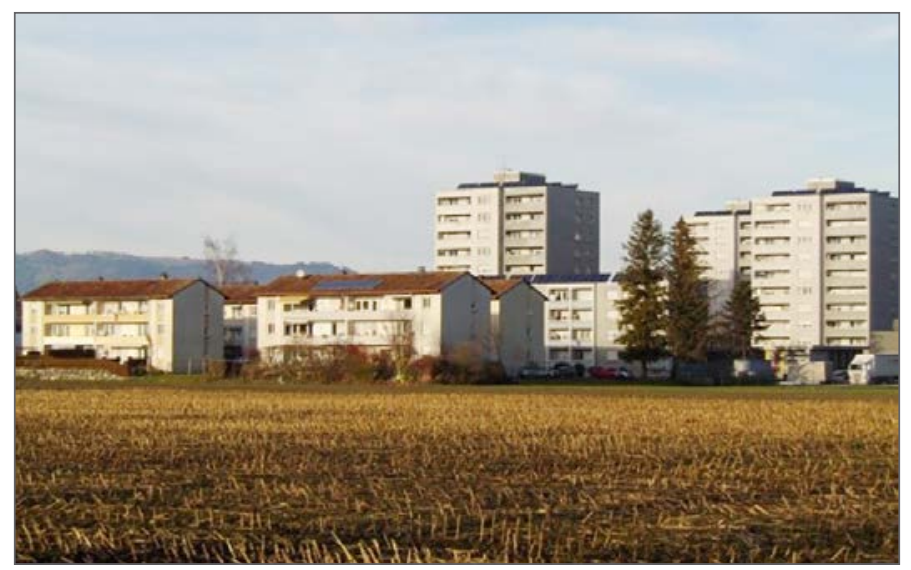

Foto: J. Herburger, 2015

Abb. 7: Gemeinnütziger Mietwohnungsbau in der Nähe des ehemaligen Areals der Textilfirma Kunert in Rankweil-Paspels 
absolventen mit 53 Prozent deutlich über dem Durchschnitt. Diese Entwicklung dürfte durch die aktuell angespannte Lage am Wohnungsmarkt nur weiter verstärkt werden.

Bei einem Blick auf die Berggemeinden wird ersichtlich, dass die einzigen Raumtypen, die sich dort finden, entweder der ländlich-landwirtschaftlich genutzte Bereich oder Einfamilienhausgebiete unterschiedlicher Generationen sind. Dies impliziert, dass die Berggemeinden haushalts- und familienstrukturell wie auch baulich noch sehr traditionell geprägt sind und von den sozialräumlichen Entwicklungen im Talbereich größtenteils (noch) nicht betroffen sind. Einzig in den Ortszentren von Fraxern, Laterns, Übersaxen, Schnifis, Röns und Düns finden sich vereinzelt Rasterzellen, die dem postsuburbanen Typus zugeordnet sind.

Insgesamt sollte deutlich geworden sein, dass in postsuburbanen räumlichen Strukturen, wie wir sie insbesondere im Vorderland oder im Walgau zwischen Bludenz und Nüziders vorfinden, spezifische sozialräumliche Besonderheiten selbst auf diesem niedrigen Aggregationsniveau in einer heterogenen „Masse“ unterzugehen drohen. Die Tatsache, dass ein solcher Raumtyp herauskristallisiert werden konnte, verdeutlicht hingegen, dass das Spezifikum dieser Siedlungsstruktur eben ihre innere Heterogenität darstellt und quasi einen Übergangsbereich von den baulich und sozial dichten Orts- und Stadtkernen hin zur sozialräumlich homogenen Suburbia darstellt. Die Dynamik dieses Bereiches zeigt sich an seinem starken Bevölkerungswachstum (8,9 \% im Vergleich zu 5 \% in der Untersuchungsregion im Zeitraum von 2001 bis 2011), wodurch sich weitergehend die sozialräumliche Komplexität dieses Raumtypus verdeutlicht. In Zukunft werden sich die Nutzungsansprüche noch weiter ausdifferenzieren, weshalb diesem Raumtyp eine hohe raumordnerische und sozialpolitische Relevanz zukommen sollte.

Daneben konnten sechs weitere, sehr unterschiedliche Raumtypen klassifiziert werden, die die postsuburbane Überprägung der Region als Ganzes verdeutlichen. Es wurde klar ersichtlich, dass die Industrialisierung und die flächenhafte Suburbanisierung der Nachkriegszeit einen wesentlichen Einfluss auf die sozialräumliche Struktur ausüben, was anhand der sozialen und ethnischen Segregationsmuster sowie an den unterschiedlichen Alterstypen der Suburbanisierungsbereiche erkannt werden konnte. Dadurch wird die generationenübergreifende raumprägende Relevanz des Einfamilienhauses verdeutlicht, was insbesondere vor dem Hintergrund des Wachstums der Siedlungsrandbereiche zu Ungunsten der älteren Suburbanisierungsbereiche als klare Problemstellung anzusprechen ist. Der Notwendigkeit, einen dementsprechend konstruktiven und aktiven Umgang mit diesem Gebäudetypus zu finden, sollte daher höchste Priorität zukommen.

Demgegenüber steht in einer solch postsuburbanen Region eine Vielzahl von Bewohnerinnen und Bewohnern, für die das Einfamilienhaus nicht (mehr) die geeignete Wohnform darstellt. Dies betrifft insbesondere die Alterung der Bevölkerung, die in jedem Raumtyp in der einen oder anderen Form vorzufinden ist. Es stellt sich daher die Frage, welche Auswirkungen die Alterung in den Suburbanisierungsbereichen für die postsuburbanen oder zentralörtlichen Gebiete hat. Dabei geht es um die Entwicklung neuer Wohnformen im Alter, die Mobilität, Selbstversorgung und Sicherung der sozialen Kontakte bis ins hohe Alter sicherstellen. So könnten gleichzeitig untergenutzte bzw. vom Leerstand bedrohte Immobilien für die Um- und Nachnutzung aktiviert werden, um dadurch den 
Druck auf die Siedlungsrandbereiche zu verringern. Ebenso müssen aber auch für junge Bevölkerungsgruppen entsprechende Wohnungsangebote geschaffen werden.

Aufgrund der weiteren Internationalisierung und der Ausdifferenzierung der Bevölkerung durch den demographischen Wandel ist es notwendig, auch ein städtebaulich integriertes Wohnungsangebot für sozial schwächere Bevölkerungsgruppen zu schaffen, um dem Entstehen von weiteren sozialräumlichen Segregationsbereichen in Zukunft entgegenzuwirken. Wie bereits angesprochen wurde, ist dies gerade in postsuburbanen Stadtregionen, deren Kernstädte nur Klein- oder Mittelstädte sind, von Bedeutung, da ein preiswertes, städtebaulich integriertes Wohnungsangebot für solche Menschen nur in geringem Ausmaß vorhanden ist. Die Konzentration in schlecht ausgestatteten und eventuell stigmatisierten Siedlungsrandlagen wirkt sich negativ auf die Integration von Personen mit Migrationshintergrund aus.

Abschließend sollte noch ein Augenmerk auf das verwendete Datenmaterial gelegt werden. Grundsätzlich hat sich gezeigt, dass sieben sinnvoll zu interpretierende Raumtypen klassifiziert werden konnten. Allerdings sollte davon Abstand genommen werden, diese als vollständig homogene Gesamtheiten zu betrachten. Vielmehr konnte die sozialräumliche Struktur der Untersuchungsregion so generalisiert werden, dass übergeordnete Problemfelder benannt und grob verortet werden können. Zwar haben sich die Rasterdatensätze als sinnvolle Basis für eine solche Untersuchung herausgestellt, dennoch ist natürlich klar, dass es sich bei den Rasterzellengrenzen nicht direkt um in der Realität wahrzunehmende Grenzen handelt.

\section{Und was nun? - Handlungsempfehlungen als Ergebnis der sozial- räumlichen Typisierung}

Gerade aufgrund der eben angesprochenen Thematik gilt es, die Handlungsempfehlungen an den gemeinsamen Problemfeldern, die aus den Raumtypen abgeleitet werden können, zu orientieren.

\section{Etablierung einer Datenbank für Flächen- und Leerstandsmanagement}

Aufgrund der zukünftigen Alterung der Bevölkerung und zur Minderung des Drucks auf die Siedlungsränder müssen aktivierende Instrumente für die Innenentwicklung der Gemeinden implementiert werden. Eine Datenbank für Flächen- und Leerstandsmanagement wäre ein mögliches Beispiel, das bereits in Bayern, Hessen und Niederösterreich umgesetzt wird.

\section{Bewerbung und Förderung alternativer Wohnformen}

Vor dem Hintergrund der angesprochenen gesellschaftlichen Entwicklungen, der mangelnden Verfügbarkeit von Bauflächen sowie des zukünftigen Generationenwechsels wird es wichtig sein, neue Wohnformen zu etablieren. „In Abgrenzung zu konventionellen Bauträgerprojekten können Baugemeinschaften, Genossenschaften oder Mietervereinslösun- 
gen sowohl räumliche Qualitäten [... ] als auch soziale Qualitäten auf einem neuen Niveau anbieten. Die Bau-, Renovierungs- und laufenden Nebenkosten sind in der Regel billiger, da nicht der externe Gewinn im Mittelpunkt steht, sondern es sich um Nutzergemeinschaften handelt, die dort selbst wohnen wollen“" (WONNEBERGER 2015, S. 21).

\section{Berücksichtigung sozialräumlicher Strukturen bei der Siedlungsentwicklung}

Neben den bereits angesprochenen Problembereichen kommt den Raumtypen „Hochverdichtete Zentren“ und „Postsuburbia“ eine besondere Stellung zu, da diese grundsätzlich als Zielgebiete für vielfältige Nutzungen anzusehen sind. Die qualitativ hochwertigere Erschließung durch den öffentlichen Personen-Nahverkehr aufgrund der höheren Dichten und die damit verbundene bessere Erreichbarkeit von Einrichtungen der Daseinsvorsorge spielen dabei eine wesentliche Rolle. Aus raumordnerischer Sicht kann durch diese Voraussetzungen die gewünschte funktionale und soziale Durchmischung erreicht werden. Aufgrund der vielfältigen Nutzungsansprüche sollten allerdings bereits bei der weiteren Siedlungsentwicklung konfliktminimierende Maßnahmen wie Partizipation, Mediation und proaktives Quartiersmanagement implementiert werden, um so die Akzeptanz für Verdichtungsmaßnahmen zu stärken.

\section{Förderung nachbarschaftlicher Beziehungen}

Allerdings stellt sich auch in den anderen Raumtypen die Frage, wie die Siedlungsstruktur nachhaltig und qualitätsvoll weiterentwickelt werden kann, ohne die nachbarschaftlichen Beziehungen zu destabilisieren, da diese insbesondere in Quartieren, die von zunehmender Alterung und sozialer Segregation betroffen sind, eine wichtige Rolle spielen. Die nachbarschaftlichen Beziehungen in suburbanen Quartieren, die von einer Überalterung der Bewohnerschaft betroffen sind, beruhen vor allem auf der Pflege von sozialen Kontakten und auf Hilfestellungen. Daher sollte der nachbarschaftlichen Integration von frisch zugezogenen Personen und Familien eine wichtige Rolle zukommen, um ebensolche sozialen Qualitäten aufrecht zu erhalten bzw. zu fördern.

\section{Siedlungsstrukturelle Anpassung an eine alternde Gesellschaft}

Neben der Bewerbung und Förderung neuer Wohnformen im Alter wird aber auch die Anpassung des Wohnungsbestandes und des Wohnumfeldes an eine zunehmende ältere Gesellschaft notwendig sein. Dabei ist insbesondere anzunehmen, dass gerade der Einfamilienhausbestand aufgrund der Wohnbausubstanz nicht den Mobilitätsbedürfnissen älterer Menschen gerecht wird.

\section{Fazit}

In der vorliegenden Arbeit wurde versucht, mittels einer quantitativen Analyse verschiedene Raumtypen in der LEADER-Region Vorderland-Walgau-Bludenz zu klassifizieren. 
Dies wurde auf einem sehr niedrigen Aggregationsniveau in Form von 250 x 250 m Rastern vorgenommen, um so kleinteilige sozialräumliche Muster und Problemstellungen herauszuarbeiten. Die theoretische Basis, die durch die Konzepte der Suburbanisierung und Postsuburbanisierung gelegt wurde, hat sich als sinnvolles Fundament zur Ableitung von 13 Indikatoren erweisen. Durch die Dimensionsreduzierung dieser Daten mittels Hauptkomponenten- und Clusteranalyse konnten sieben sozialräumliche Raumtypen herausgefiltert werden, die verschiedene Entwicklungshintergründe und Problemstellungen aufweisen, die oftmals nicht den Zielsetzungen der Raumordnung entsprechen. In den Handlungsempfehlungen wurden daher alternative Instrumente und Methoden vorgestellt, an welchen sich die LEADER-Region orientieren kann.

Anhand der gewonnen Ergebnisse hat sich gezeigt, wie sehr die baulich-räumliche Struktur mit unterschiedlichsten sozio-demographischen, sozio-kulturellen und sozio-ökonomischen Faktoren in Verbindung steht, die zusammen spezifische Problemstellungen für Politik, Verwaltung und Gesellschaft bedeuten. Der sozialräumlich polarisierenden Wirkung des Wohnungsmarktes sollte daher ein hohes Augenmerk geschenkt werden. Dabei ist insbesondere zu bedenken, dass die Prozesse der gesellschaftlichen Pluralisierung, Alterung und Migration nicht zu stoppen sind, wodurch sich die Raumstruktur in Zukunft weiter ausdifferenzieren wird. Es ist daher von großer Bedeutung, neue Wege auf dem Wohnungsmarkt zu gehen, um der sozialen Segregation sowie der Randwanderung der Bevölkerung entgegenzuwirken. Das bedeutet, dass es möglich sein muss, in städtebaulich sinnvollen Lagen unter Berücksichtigung des sozialräumlichen Umfeldes bedarfsgerechten Wohnraum für alle Gruppen unserer Gesellschaft zu schaffen.

Gleichsam als räumliches Ergebnis der Pluralisierung der Gesellschaft ist die Postsuburbia zu betrachten, die sich selbst bei einer solch hohen Datenauflösung als insgesamt heterogener Raumtyp zwischen Stadt und Einfamilienhausgebiet herauskristallisiert hat. Auf regionaler Ebene zeigt sich hingegen die Fragmentierung der Raumstruktur durch Raumtypen, die unterschiedliche sozialräumliche Problemfelder beinhalten und von der Segregation bestimmter Bevölkerungsgruppen über die klassischen Einfamilienhausgebiete bis hin zu ebendieser postsuburbanen Raumstruktur führen. Allerdings führte die Industrialisierung in der LEADER-Region im Zusammenhang mit dem gemeinnützigen, hochverdichteten Mietwohnungsbau schon früh zu internationalen Zuwanderungsbewegungen, wodurch die sozialräumliche Struktur bis heute beeinflusst wird. Daher kann auch die gesamte Region als postsuburbaner Raum eingestuft werden, wenngleich insbesondere die Berggemeinden noch sehr traditionelle sozialräumliche Muster aufweisen.

Die vorliegende Analyse verdeutlicht also, dass die Entstehung von postsuburbanen siedlungs- und sozialräumlichen Strukturen, wie sie vorwiegend im Umland von Großstädten zu finden sind, eben nicht auf diese Räume beschränkt ist. Die Megaprozesse, die insbesondere seit dem Ende des Zweiten Weltkriegs unsere gesamte europäische Siedlungsstruktur verändert und überprägt haben, wirken sich in ähnlicher Form auch auf Räume ohne Kernstädte aus. Die Alpen im Allgemeinen und auch die Untersuchungsregion als wirtschaftlich prosperierende Region im Spezifischen haben sich als passender räumlicher Rahmen dafür dargestellt. Die Postsuburbanisierung als spezifische Form globaler Urbanisierungsbewegungen und die damit einhergehenden Planungsherausforderungen zeichnen sich als mannigfaltig aus und betreffen neben der Raumordnung selbst noch zahlreiche andere Fachpolitiken. 


\section{Literaturverzeichnis}

ARING J. (2001): Suburbia - Postsuburbia - Zwischenstadt. Ältere und neuere Entwicklungstrends am Rande der Großstadt. Arbeitspapier zum Vortrag in Garbsen am 10.11.2000.

Aring J., Herfert G. (2001): Neue Muster der Wohnsuburbanisierung. In: Brake K., Dangschat J. S., Herfert G. (Hrsg.): Suburbanisierung in Deutschland - Aktuelle Tendenzen. Opladen: Leske + Budrich, S. 43-56.

Backhaus K., Erichson B., Plinke W., Weiber R. ( ${ }^{51990): ~ M u l t i v a r i a t e ~ A n a l y s e m e t h o d e n ~-~ E i n e ~}$ anwendungsorientierte Einführung. - Berlin / Heidelberg: Springer.

BäHR J. ( $\left.{ }^{5} 2010\right)$ : Bevölkerungsgeographie. Verteilung und Dynamik der Bevölkerung in globaler, nationaler und regionaler Sicht. Stuttgart: Ulmer (= UTB Uni-Taschenbücher, 1249).

Bahrenberg G., Giese E., NipPer J. ( $\left.{ }^{2} 1992\right)$ : Statistische Methoden in der Geographie - Band 2: Multivariate Statistik. Stuttgart: Teubner (Teubner Studienbücher der Geographie).

BäTzING W. ( $\left.{ }^{4} 2015\right)$ : Die Alpen - Geschichte und Zukunft einer europäischen Kulturlandschaft. München: C. H. Beck.

Bleyle A. (о. J.): Entstehung und Entwicklung der Vorarlberger Industrie. - https://wirtschaftsarchiv -v.at/files/Industrie.pdf (Zugriff: 27.11.2018).

BorSDORF A. (2006): Land-Stadt Entwicklung in den Alpen - Dorf oder Metropolis? In: PsenNer R., LACKNER R. (Hrsg.): Die Alpen im Jahr 2020. Innsbruck: Innsbruck University Press, S. 83-93 (= Alpine Space - Man \& Environment, 1).

BorSDORF A. (2009): Schlaraffenland oder Niemandsland. Suburbanisierung - ein problematischer Boom? In: Ländlicher Raum - Online-Fachzeitschrift des Bundesministeriums für Landund Forstwirtschaft, Umwelt und Wasserwirtschaft, Jg. 2009, S. 1-21.

Borsdorf A., Bender O. (2010): Allgemeine Siedlungsgeographie. Wien / Köln / Weimar: Böhlau (UTB Uni-Taschenbücher, 3396).

Brake K., Einacker I., MäDing H. (2005): Kräfte, Prozesse, Akteure. Zur Empirie der Zwischenstadt. Wuppertal: Müller \& Busmann (= Schriftenreihe Zwischenstadt, 3).

BüLöw R. (1996): Faktoren- und Clusteranalyse - Zwei multivariate statistische Analyseverfahren am Beispiel der Hauptkomponentenanalyse des Clusterverfahrens nach Ward und der k-means-Methode -Eine anwenderorientierte Einführung mit SPSS anhand zweier idealtypischer beispiele. Bochum: Fakultät für Sozialwissenschaften, Ruhr-Universität Bochum.

BURTSCHER S. (2009: Zuwandern - aufsteigen - dazugehören. Etablierungsprozesse von Eingewanderten. Innsbruck: StudienVerlag (= Transblick, Sozialwissenschaftliche Reihe, 4).

Fassmann H. (2009): Stadtgeographie I. Allgemeine Stadtgeographie. Braunschweig: Westermann (Das Geographische Seminar).

FRIEDRICHS J. ( $\left.{ }^{3} 1983\right)$ : Stadtanalyse: Soziale und räumliche Organisation der Gesellschaft. Opladen: Westdeutscher Verlag.

Görgl P. J. (2008): Die Amerikanisierung der Wiener Suburbia? Der Wohnpark Fontana - Eine sozialgeographische Studie. Wiesbaden: VS Verlag für Sozialwissenschaften.

Gutfleisch R. (2007): Sozialräumliche Differenzierung und Typisierung städtischer Räume - Ein Methodenvergleich am Beispiel der Stadt Frankfurt am Main. Frankfurt am Main: Institut für Humangeographie, Universität Frankfurt am Main (= Rhein-Mainische Forschungen, 128).

Helbich M. (2008): Modellierung (post)suburbaner Prozesse am Fallbeispiel der Stadtregion Wien. Dissertation an der Universität Wien (2009 unter dem gleichen Titel publiziert im Lit-Verlag, Berlin / Münster / Wien, Reihe „Beiträge zur Europäischen Stadt- und Regionalforschung, 7).

HöPFLINGER F. (1997): Bevölkerungssoziologie. Eine Einführung in bevölkerungssoziologische Ansätze und demographische Prozesse. Weinheim / München: Juventa. 
Kunzmann K. R. (2001): Welche Zukünfte für Suburbia? Acht Inseln im Archipel der Stadtregion. - In: Brake K., Dangschat J. S., Herfert G. (Hrsg.): Suburbanisierung in Deutschland Aktuelle Tendenzen. Opladen: Leske + Budrich, S. 213-221.

Knapp W., Volgmann K. (2011): Neue ökonomische Kerne in nordrhein-westfälischen Stadtregionen: Postsuburbanisierung und Restrukturierung kernstädtischer Räume. In: Raumforschung und Raumordnung, 69 (4), S. 303-317.

LAG [Lokale Arbeitsgruppe] Vorderland-Walgau-Bludenz (2014): Lokale Entwicklungsstrategie 2014-2020. - https://www.vorderland.com/medien/leader_entwicklungsstrategie-final.pdf (Zugriff: 27.11.2018)

Lesthaeghe R. (2011): The "Second Demographic Transition": A Conceptual Map for the Understanding of Late Modern Demographic Developments in Fertility and Family Formation. In: Historical Social Research, 36 (2), S. 179-218.

Lukesch R., PAyer H., Winkler-Rieder W. (2010): Wie gehen Regionen mit Krisen um? - Eine explorative Studie über die Resilienz von Regionen. Fehring: ÖAR-Regionalberatung GmbH.

Norusis M. J. (1993): SPSS for Windows - Professional Statistics, Release 6.0. Englewood Cliffs / Chicago: SPSS Inc.

Nuissl H., Bigalke B. (2007): Altwerden am Stadtrand - zum Zusammenhang von Suburbanisierung und demographischem Wandel. In: Raumforschung und Raumordnung, 65 (5), S.,381-392.

PerLiK M. (1999): Alpen, Städte und Europa. Die Alpenstädte als Teil eines europäischen Städtesystems. In: Revue de géographie alpine, 87 (2), S. 23-36.

Perlik M. (2001): Alpenstädte - Zwischen Metropolisation und neuer Eigenständigkeit. Bern: Geographisches Institut Bern (= Geographica Bernensia, Serie P, 38).

Perlik M., Messerli P., Bätzing W. (2001): Towns in the Alps. In: Mountain Research and Development, 21 (3), S. 243-252.

PoHL T. (2010): Folgen des demographischen Wandels in einer „Gewinnerregion“. Kleinräumig differenzierte Betrachtung der Bevölkerungsentwicklung in der ,wachsenden Stadt“ Hamburg. In: Raumforschung und Raumordnung, 68 (3), S. 195-206.

Prigge W. (1998): Vier Fragen zur Auflösung der Städte. In: Prigge W. (Hrsg.): Peripherie ist überall. Frankfurt am Main: Campus, S. 8-15 (= Edition Bauhaus, 1).

Saiko H. (2003): Ideales Wohnen - Ein Europa der Einfamilienhäuser. In: Hubeli E., SAiko H., VöCKLER K. (Hrsg.): 100 \% Stadt - Der Abschied vom Nicht-Städtischen. Graz: Verlag Haus der Architektur, S. 101-108.

SchwANZer W. (1987): Suburbanisierung im Main-Kinzig-Kreis - Ein Beitrag zum Problem des Sozialraumwandels. Frankfurt am Main: Institut für Humangeographie, Universität Frankfurt am Main (= Rhein-Mainische Forschungen, 104).

Spellerberg A., Gerhards P. (2013): Lebensbedingungen und Wohnwünsche älterer Menschen Ergebnisse aus der Stadt Pirmasens. In: Junkernheinrich, M., Ziegler K. (Hrsg.): Räume im Wandel - Empirie und Politik. Wiesbaden: Springer, S. 119-140.

Trossbach W., Zimmermann C. (2006): Die Geschichte des Dorfes: Von den Anfängen im Frankenreich zur bundesdeutschen Gegenwart. Stuttgart: Ulmer (= UTB Uni-Taschenbücher 8324).

WonNEBERGER E. (2015): Neue Wohnformen - Neue Lust am Gemeinsinn? Wiesbaden: VS Verlag für Sozialwissenschaften.

ZöFEL P. (2002): Statistik verstehen - Ein Begleitbuch zur computergestützten Anwendung. München / Boston: Addison-Wesley. 